\title{
Whole-genome profiling of nasopharyngeal carcinoma reveals viral-host co-operation in inflammatory NF- $\kappa B$ activation and immune escape
}

Jeff P. Bruce ${ }^{1,15}$, Ka-Fai To (1) 2,3,15, Vivian W. Y. Lui (10 4,15, Grace T. Y. Chung 2,15, Yuk-Yu Chan², Chi Man Tsang², Kevin Y. Yip (1) ${ }^{5}$, Brigette B. Y. Ma(i) ${ }^{3,6,7}$, John K. S. Woo ${ }^{8}$, Edwin P. Hui (1) ${ }^{3,6,7}$, Michael K. F. Mak ${ }^{2}$, Sau-Dan Lee ${ }^{5}$, Chit Chow (i) ${ }^{2}$, Sharmila Velapasamy ${ }^{2}$, Yvonne Y. Y. Or ${ }^{2}$, Pui Kei Siu ${ }^{2}$, Samah El Ghamrasni (1) ${ }^{1}$, Stephenie Prokopec (D) ${ }^{1}$, Man Wu ${ }^{2}$, Johnny S. H. Kwan², Yuchen Liu (D) ${ }^{4}$, Jason Y. K. Chan (D) ${ }^{8}$, C. Andrew van Hasselt ${ }^{8}$, Lawrence S. Young $~^{9}{ }^{9}$, Christopher W. Dawson ${ }^{9}$, Ian C. Paterson (1) ${ }^{10}$, Lee-Fah Yap (iD ${ }^{10}$, Sai-Wah Tsao ${ }^{11}$, Fei-Fei Liu'1,12, Anthony T. C. Chan ${ }^{5}$, Trevor J. Pugh (1) ${ }^{1,13,14 凶 \&} \&$ Kwok-Wai Lo id 2,3凶

Interplay between EBV infection and acquired genetic alterations during nasopharyngeal carcinoma (NPC) development remains vague. Here we report a comprehensive genomic analysis of 70 NPCs, combining whole-genome sequencing (WGS) of microdissected tumor cells with EBV oncogene expression to reveal multiple aspects of cellular-viral co-operation in tumorigenesis. Genomic aberrations along with EBV-encoded LMP1 expression underpin constitutive NF- $\mathrm{kB}$ activation in $90 \%$ of NPCs. A similar spectrum of somatic aberrations and viral gene expression undermine innate immunity in $79 \%$ of cases and adaptive immunity in $47 \%$ of cases; mechanisms by which NPC may evade immune surveillance despite its proinflammatory phenotype. Additionally, genomic changes impairing TGFBR2 promote oncogenesis and stabilize EBV infection in tumor cells. Fine-mapping of CDKN2A/CDKN2B deletion breakpoints reveals homozygous MTAP deletions in $32-34 \%$ of NPCs that confer marked sensitivity to MAT2A inhibition. Our work concludes that NPC is a homogeneously NF-kBdriven and immune-protected, yet potentially druggable, cancer.

\footnotetext{
${ }^{1}$ Princess Margaret Cancer Centre, University Health Network, Toronto, ON, Canada. ${ }^{2}$ Department of Anatomical and cellular Pathology, The Chinese University of Hong Kong, Hong Kong SAR, China. ${ }^{3}$ State Key Laboratory of Translational Oncology, The Chinese University of Hong Kong, Hong Kong SAR, China. ${ }^{4}$ School of Biomedical Sciences, The Chinese University of Hong Kong, Hong Kong SAR, China. ${ }^{5}$ Department of Computer Science and Engineering, The Chinese University of Hong Kong, Hong Kong SAR, China. ${ }^{6}$ Sir Y.K. Pao Centre for Cancer, The Chinese University of Hong Kong, Hong Kong SAR, China. ${ }^{7}$ Department of Clinical Oncology, The Chinese University of Hong Kong, Hong Kong SAR, China. ${ }^{8}$ Department of Otorhinolaryngology, Head and Neck Surgery, Prince of Wales Hospital, The Chinese University of Hong Kong, Hong Kong SAR, China. ${ }^{9}$ Warwick Medical School, University of Warwick, Coventry, UK. ${ }^{10}$ Department of Oral \& Craniofacial Sciences and Oral Cancer Research and Coordinating Centre, University of Malaya, Kuala Lumpur, Malaysia. ${ }^{11}$ School of Biomedical Sciences, Li Ka Shing Faculty of Medicine, The University of Hong Kong, Hong Kong SAR, China. ${ }^{12}$ Department of Radiation Oncology, University of Toronto, Toronto, ON, Canada. ${ }^{13}$ Department of Medical Biophysics, University of Toronto, Toronto, ON, Canada. ${ }^{14}$ Ontario Institute for Cancer Research, Toronto, ON, Canada.

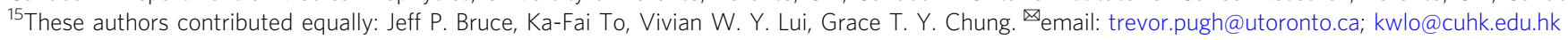


$\mathrm{N}$ asopharyngeal carcinoma (NPC) is an etiologically complex tumor with a unique combination of epidemiologic, histologic, and viral features. NPC afflicts $\sim 129,000$ new patients each year (GLOBOCAN 2018), with the highest incidence occurring in South-East Asia, North Africa, and in the Inuit population in the northern regions of North America ${ }^{1-3}$. NPC is characterized by poorly differentiated malignant epithelial cells residing in a complex microenvironment with heavy lymphocyte infiltration, giving rise to the appearance of a lymphoepithelioma with an apparent inflammatory phenotype. Malignant cells are uniformly positive for the Epstein-Barr virus (EBV), a herpesvirus widespread in the human population. EBV persistent latent infection of B lymphocytes is causally linked to several Bcell malignancies including Burkitt lymphoma, Hodgkin disease, AIDS-related lymphoma, diffuse large B-cell lymphoma, along with a somewhat unexpected association with epithelial tumors such as NPC and a small subset of gastric carcinomas.

Recent functional and genomic studies have implicated NF- $\kappa \mathrm{B}$ pathway activation (e.g., TNFAIP3, MYD88) and immune evasion (e.g., PDL1/CD274, PDL2/PDCD1LG2) in the pathogenesis of EBV-associated lymphomas ${ }^{4-7}$. However, there remains little understanding of how somatic genetic aberrations and viral infection co-operate in the malignant process. In a paradox particular to NPC, it is unclear how viral and somatic factors may interact to establish the tumor's inflammatory phenotype while rendering the malignant cells apparently obscured from detection by host immunity. Furthermore, the consistent presence of EBV in NPC is puzzling given that the epithelial cells from which NPC derives do not normally sustain a latent virus infection ${ }^{3,4}$. This raises a long-standing question as to whether NPC development involves cellular genetic changes that favor EBV persistence.

As an immediate background to the present study, wholeexome, genome, and targeted DNA-sequencing studies of primary and recurrent NPCs have revealed various somatic alterations that drive constitutive nuclear factor kappa B (NF- $\kappa \mathrm{B})$ signaling activation in up to $40 \%$ of cases ${ }^{8-14}$. Our previous work, in this context, isolating the malignant NPC cells from their abundant lymphocytic infiltrate by microdissection, used exome sequencing to identify two driver genes in the NF- $\kappa \mathrm{B}$ pathway, $T R A F 3$ and $C Y L D^{8}$. Both genes are frequently inactivated by focal homozygous deletions or rearrangements, highlighting the importance of large chromosomal aberrations or structural variants (SVs) in NPC development. The complex nature of genomic changes in NPC underscored the need for whole-genome characterization in conjunction with the evaluation of EBV gene expression, to provide further insights into the interplay of somatic aberrations and viral effects in disease pathogenesis.

In this work, we employed whole-genome sequencing (WGS) to comprehensively delineate the genome landscape of 63 microdissected tumors, 5 patient-derived xenografts, and 2 cell lines of NPC. This included analysis of etiology-associated coding and non-coding mutational signatures, detection of recurrent SVs, and fine mapping of chromosome- and gene-level copy changes across the genome. This approach illuminated multiple routes to constitutive NF- $\mathrm{kB}$ activation, as well as immune evasion mechanisms, both involving somatic changes and viral factors. We also uncovered a prevalent mutational signature associated with homologous recombination (HR) repair defects in NPC, revealing a prominent involvement of double-stranded DNA repair defects in NPC pathogenesis. Resulting structural alterations implicate two targets with critical impacts: recurrent TGFBR2 inactivation that promotes EBV persistence in tumor cells and MTAP deletion (adjacent to the CDKN2A/CDKN2B loci) with marked sensitivity to MAT2A inhibition potentially applicable to a broad subset of NPC patients. Our findings characterize NPC as universally driven by pro-inflammatory
NF- $\kappa \mathrm{B}$ signaling counterbalanced by multiple mechanisms of immune protection.

\section{Results}

Mutational signatures and significantly mutated genes. To establish a comprehensive catalog of genomic aberrations in NPC, we conducted WGS of 63 EBV-positive, non-keratinizing microdissected tumors from Southern Chinese patients (58 primary, 4 recurrent, 1 metastatic; Supplementary data 1), as well as 7 recently established cell lines and PDX models ${ }^{15}$. We achieved genome-wide average sequence coverage of $83 \times$ for tumors and $51 \times$ for paired normal controls. WGS enabled identification of both coding $(n=54,588)$ and non-coding $(n=885,442)$ simple somatic mutations, which include single nucleotide variants (SNVs), small insertions, and deletions (indels) (Supplementary Data 2, Supplementary figure 1). An orthogonal validation rate of $96 \%(82 / 85$ events with $>10 \%$ allelic-fraction, Supplementary data 3) was achieved by target-capture deep sequencing.

To provide clues regarding NPC etiology, we examined the single base changes observed which confirmed that the predominant type of genome-wide substitution is $\mathrm{C}>\mathrm{T}$ transitions at $\mathrm{NpCpG}$ sites. We also observed recurrent evidence of Signature 3 (present in $64.3 \%(45 / 70)$ cases), which was not reported by previous exome-sequencing studies ${ }^{8-10}$. Mutational Signature 3 is associated with defects in DNA double-strand break repair by HR (Fig. 1A). In addition to HR defects (Signature 3), we found contributions from deamination of 5-methyl-cytosine (Signature 1, 98.6\% cases), defective DNA mismatch repair (Signatures 6, 15, 20, and 26, 98.6\% cases) and APOBEC/AID (Signatures 2 and 13, $44.3 \%$ ). Together, these mutational signatures provide insight into NPC development that may underlie the acquisition of specific driver mutations ${ }^{8-10}$. In a recent meta-analysis study of the published exome-sequencing data sets in 402 NPC patients, all four mutational signature classes we identified were confirmed ${ }^{16}$.

To nominate coding and non-coding mutations that drive NPC, we employed ActiveDriverWGS. This identified 11 significantly mutated coding genes and one regulatory region that largely converge on NF- $\kappa \mathrm{B}$ signaling (Fig. 1B). Consistent with previous studies, TP53 was the most significantly mutated gene $\left(n=10 ; q=1.1 \times 10^{-6}\right)$, followed by TRAF3, NFKBIA, AEBP1, and NLRC5; all of which have been reported to regulate NF- $\kappa B$ signaling $4,8-14,17$. In addition, significant somatic aberrations detected in HLA-A and NLRC5 suggest impairment of antigen presentation while PTEN mutations may activate the PI3K pathway ${ }^{8-10}$. We also found four coding genes, which were significantly mutated, namely PLIN4, MUC21, SLC35G5, and $E R V W-1$. Further studies would be required to define the function of these mutations in NPC.

Among all the SNVs and indels detected, 94\% were located in non-coding regions. These include 1480 mutations in putative promoters (The Eukaryotic Promoter Database), 5997 in predicted Enhancers (ENCODE), and 4699 in TF-binding sites (ENCODE). The sole significantly mutated non-coding regulatory region in our cohort, ENSR00000289706, is a CTCF binding site $\sim 16 \mathrm{~kb}$ downstream from CYS1 and $\sim 22 \mathrm{~kb}$ upstream of RRM2 (Fig. 1B). Notably, a single recurrent mutation (chr2:10097565 C > T) was identified in four tumors from three patients (Supplementary figure 2). Despite reports from several genome sequencing studies conducted thus far, this represents the first recurrent mutation identified in the non-coding region of NPC genome $e^{8-14}$. Furthermore, this mutation results in a gain of a putative NFKB1/p50 binding sequence upstream of $R R M 2$ which has been reported to be associated with NF- $\mathrm{BB}$-signaling activation $^{18}$. Of note, mutations in this region were also reported in the International Cancer Genome Consortium (ICGC) 


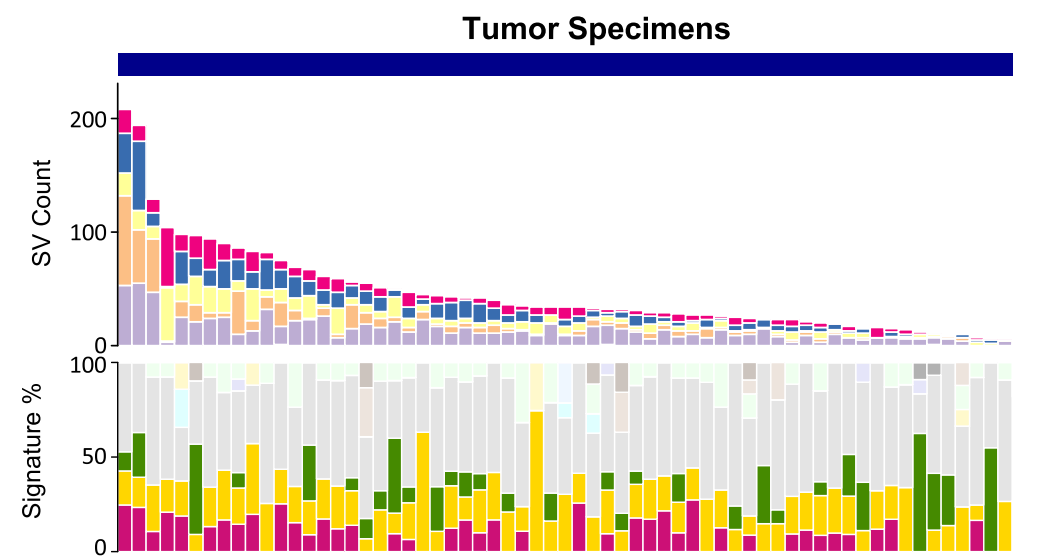

Cell lines

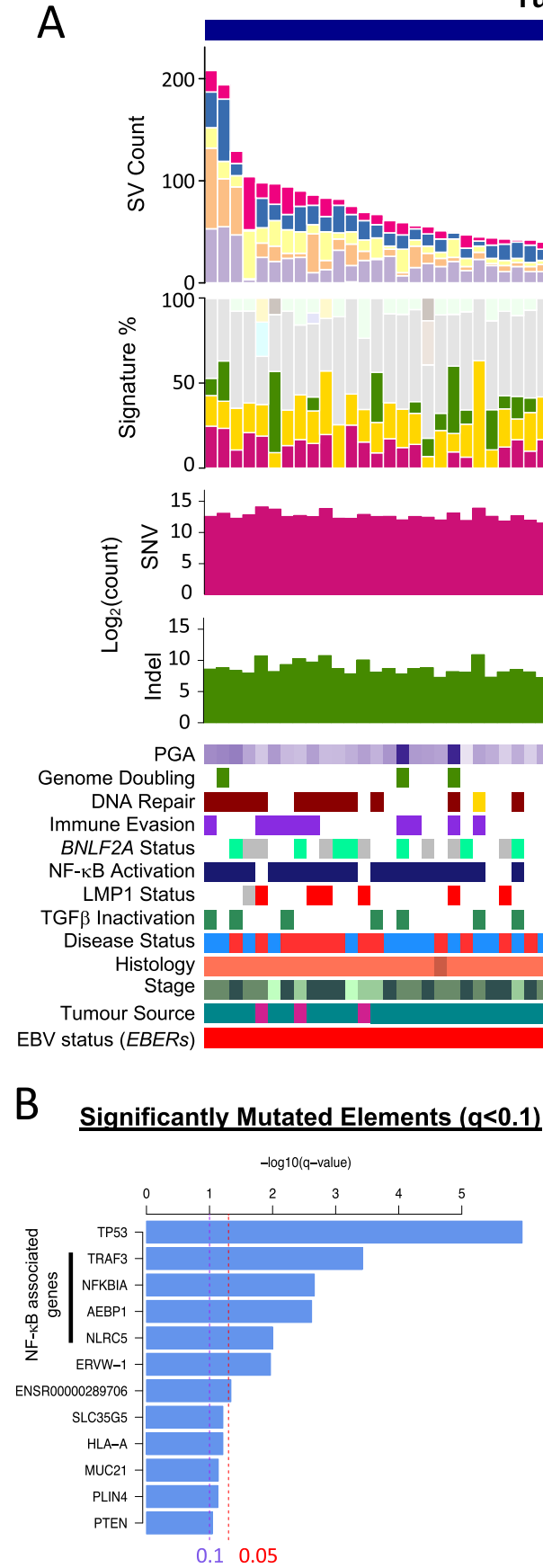

\section{\& PDXs}
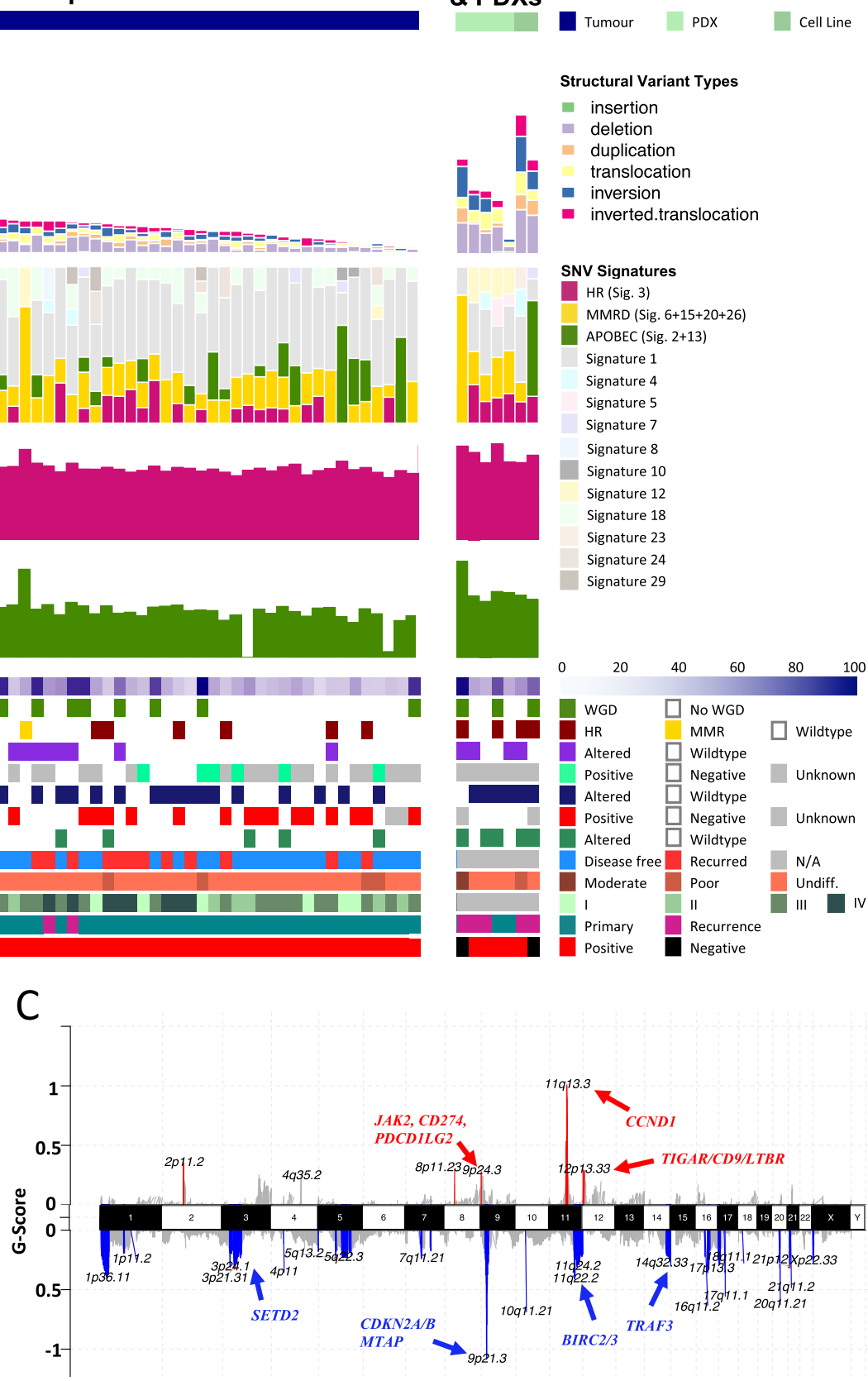

Fig. 1 Whole-genome landscape of NPC. A Somatic gene alterations and mutational signatures of 70 NPC tumors. For each tumor, the number and types of SNVs and SVs, mutation signatures, whole-genome doubling, and percent genome altered (PGA) were shown in the top panels, as well as somatic changes detected in NF-KB, DNA repair, and TGF- $\beta$ pathways, expression of EBV-encoded LMP1, BNLF2a and EBERs, clinical staging and tumor type. B Significantly mutated genes and regulatory elements $(q<0.1)$ identified in NPC. The altered genes in NF-kB pathways are indicated. C Copy number alterations in NPC. GISTIC copy number variations analysis showing recurrent amplification and deletion of multiple chromosomal regions.

pan-cancer data set, including three occurrences of this specific point mutation ${ }^{19}$. Future studies would be required to confirm the predicted functional effect of this and other, less-frequent non-coding mutations.

Recurrent copy number and structural alterations. We identified somatic copy number alterations (SCNAs) using Varscan2 and Sequenza, followed by significance analysis using GISTIC (v2.0.23) (Fig. 1C, supplementary data 4). We detected polyploidy in $15.9 \%$ of patient tumors $(10 / 63)$ and the median percent genome altered (PGA) was 30\% (range 9-92\%) (Fig. 1C). GISTIC analysis revealed frequent arm-level chromosomal losses and amplification, including frequent gains in chr.1q, 3q, 7q, 8q, 12p, and 12q, and frequent losses in chr. 3p, 9p, 11q, 14q, and 16q (Supplementary figure 3). This analysis defined homozygous deletion of $9 \mathrm{p} 21.3$ (CDKN2A/CDKN2B) as the most frequently altered chromosome region, impacting $37.1 \%$ of cases (Fig. 2) 2 $^{1,2}$. 


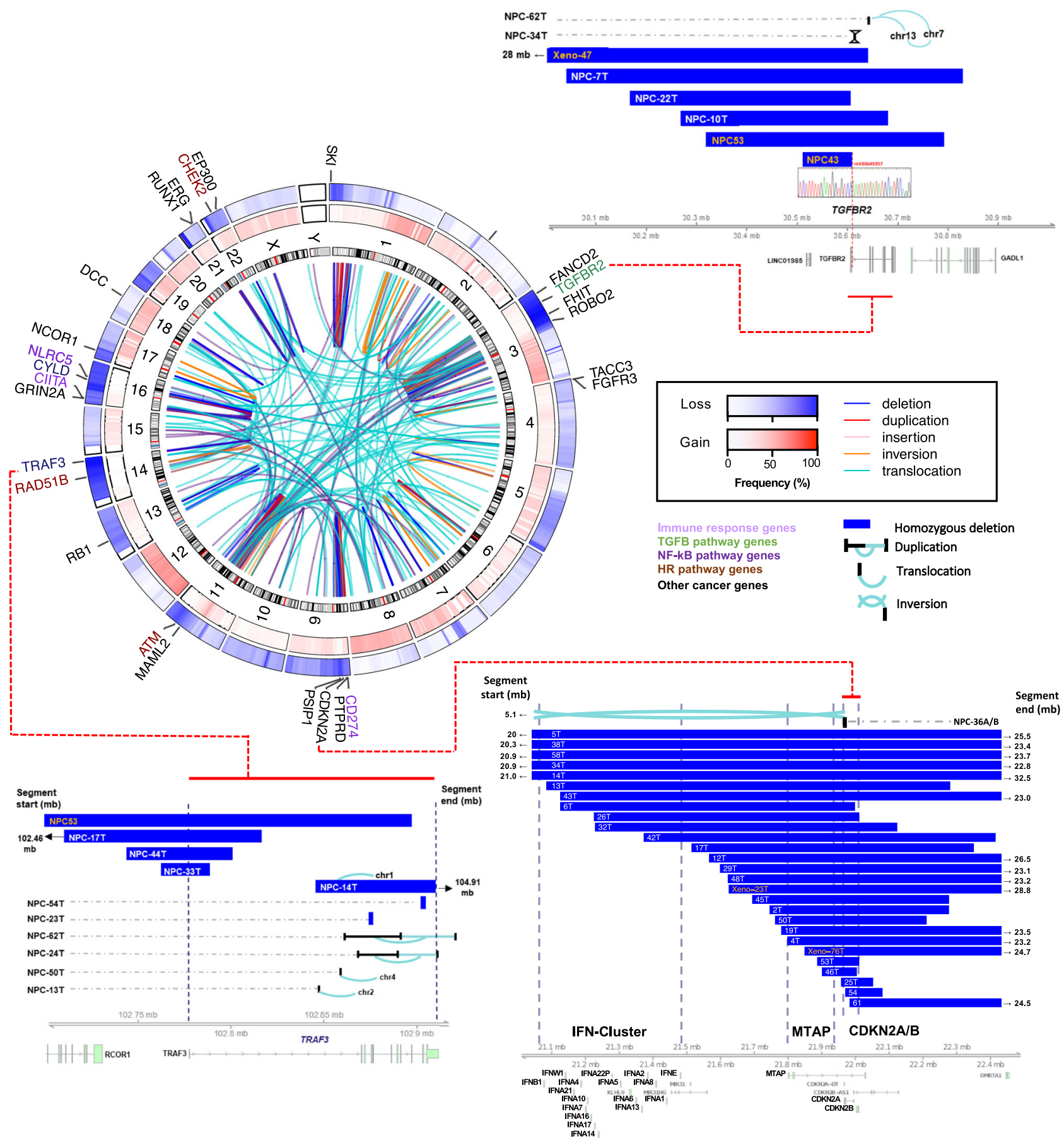

Fig. 2 Recurrent structural and chromosomal alterations in NPC. Circos plot showing recurrent SVs and common CNVs in NPC. Frequencies of copy number losses and gains are shown in the outer and inner circles respectively. Selected cancer genes involved in the recurrent inter- and intrachromosomal SVs (i.e., genes with $\geq 3$ times altered by SVs) are indicated. Homozygous deletions and structural alteration breakpoints identified in CDKN2A/B loci, MTAP, and cluster of type I IFN genes at 9p13.3, TGFBR2 at 3p24.1, and TRAF3 at 14q32.3 are shown. The DNA sequences spanning the breakpoints of deletion of TGFBR2 identified in NPC43 and Xeno-47 were confirmed by Sanger DNA sequencing.

Through the fine mapping of deletion breakpoints within 9p21.3, we noted frequent homozygous deletions of a potential druggable target, MTAP (34\%), and distally, loss of type I interferon genes (e.g., IFNA1, IFNA2, IFNA8, IFNE; 16\%) that may be associated with loss of antiviral response in early $\mathrm{NPC}^{2,3}$ (Fig. 2). In addition, significantly focal amplified regions harboring potential drivers were found on chr. 11q13 (CCND1), 9p24.3 (JAK2, CD274, PDCD1LG2) and 12p13.3 (TIGAR/CD9/LTBR Fig. 1C). Together, these recurrent SCNAs are consistent with the previous genome-wide studies and accurately defined NPC-associated genes including CD274 and the type I interferon genes $2,8,20$. Importantly, using microdissected tumor samples and WGS, we detected the highest frequencies of homozygous deletions of the critical tumor suppressor genes CDKN2A, TGFBR2, TRAF3, and potential synthetic lethal target MTAP amongst NPC genomic reports thus arr $^{8-14}$.

Chromosomal rearrangements were detected using a combination of three SV calling algorithms (Manta (v1.2.2), DELLY (v0.7.7), NovoBreak (v1.1.3)) followed by filtration and annotation using MAVIS (v1.8.4) (Supplementary data 5). Using this 
approach, we identified a total of 3486 high-confidence SVs, including translocations, inversions, inverted translocations, insertions, deletions, and duplications (Fig. 1A). On average, NPCs harbored 50 SVs per tumor (ranging from 5 to 208), consistent with previously reported rates in head and neck squamous cell carcinoma, lung and colorectal adenocarcinomas (Fig. 1A, suppl. Figure S1). Mapping of recurrent SVs with common chromosomal aberrations revealed substantial overlap with recurrent CNAs including multiple regions on chromosome $3 p, 9 p, 11 q, 14 q$, and 16q, harboring known tumor suppressors and cancer genes in NPC (Fig. 2). While the heterozygous loss of chr $3 p$ is a known feature of NPC (64/70, 91.4\% in our cohort), we also discovered recurrent homozygous deletions and SVs of TGFBR2 at 3 p 24.1 in $11.4 \%$ of our tumors (Fig. 2). In addition, recurrent breakpoints of SVs targeting cancer genes commonly occurred in frequently deleted regions, e.g., CDKN2A on 9p21.3, $M A M L 2$ and $A T M$ on $11 \mathrm{q} 21-22, R A D 51 B$, and TRAF3 on $14 \mathrm{q} 24-$ 32.3, CYLD and NLRC5 on 16q12.1-13 (Fig. 2). Taken together, we conclude that combinations of homozygous deletions and structural alterations are common mechanisms for biallelic inactivation of tumor suppressors in NPC, highlighting the need for comprehensive genome-wide profiling for this cancer type, in particular. Consistent with previous reports, recurrent oncogenic TACC3-FGFR3 fusions were also detected in our NPC cohort (2/ $70,2.9 \%)^{2}$.

EBV and somatic changes co-operate to sustain NF- $\mathrm{kB}$ activation. By integrating all $\mathrm{SNV}, \mathrm{SV}$, and $\mathrm{CNV}$ data, we have established a comprehensive catalog of genomic changes implicating multiple oncogenic pathways in NPC. The most prevalently altered pathway encodes NF- $\kappa B$ signaling $(44 / 70 ; 62.9 \%)$ (Figs. $1 \mathrm{~A}$ and $3 \mathrm{~A}$ ). In addition to our previously reported somatic changes of NF- $\mathrm{BB}$-negative regulators [TRAF3 (21.4\%), CYLD (11.4\%), NFKBIA (10\%), NLRC5 (8.6\%), and TNFAIP3 (5.7\%)], we have identified additional alterations impacting this pathway including amplifications of LTBR (20\%) and recurrent homozygous deletions and rearrangements of BIRC2 (5.7\%) and BIRC3 (8.6\%). In particular, $L T B R$ amplification that went unreported in our previous exome study is a demonstrated driver for NF- $\mathrm{BB}$ activation in NPC and multiple myeloma ${ }^{11,20-22}$.

The EBV oncoprotein, LMP1, has been demonstrated to drive $N F-\kappa B$ pathway activation in NPC, via tumor necrosis factor receptor/TRAF3 interactions ${ }^{3}$. In this cohort, high LMP1 expression was found in $32.8 \%(23 / 70)$ of tumors (Fig. 3A, B). Previously, we reported the discovery of mutual exclusivity between somatic and LMP1-driven NF- $\kappa \mathrm{B}$-activating events in NPC which we have confirmed herein (Fig. 3B; two-sided Fisher's exact test $\left.p=3.5 \times 10^{-6}\right)$. With additional genome variations, our current WGS study further strengthened this finding, as coselected NF- $\kappa$ B-activating mechanisms in $90 \%(63 / 70)$ of this tumor cohort with a high degree of mutual exclusivity (two-sided Fisher's exact test $p=7.4 \times 10^{-7}$ ). Our findings solidly define $\mathrm{NPC}$ as a ubiquitously NF- $\mathrm{BB}$-driven malignancy. In addition, LMP1 is also known to activate PI3K/AKT and MAPK signaling pathways ${ }^{3}$. Although rare somatic alterations of PI3K pathways were observed in NPC cases with LMP1 overexpression, similar viral-somatic mutual exclusivity was observed between altered PI3K signaling pathway and LMP1 expression (Supplementary figure $4 \mathrm{~A}, \mathrm{~B})$.

Impaired immune machinery by EBV and somatic changes. With inflammatory NF- $\kappa \mathrm{B}$ signaling, persistent expression of various viral RNAs (EBERs) and immune antigens (e.g., EBNA1, LMP1, LMP2) in nearly all NPC, immune evasion is believed to be critical for NPC pathogenesis. The abundantly expressed EBER transcripts are known to activate IRF3 signaling, which induces type I interferons to elicit innate immunity against EBV-infected NPC cells ${ }^{23-25}$. Indeed, using WGS we uncovered genomic losses of multiple types I interferon genes, including INFA1, IFNA2, IFNA8, and IFNE by a homozygous deletion in $15.7 \%$ of NPC (Fig. 2). Importantly, the inactivation of TRAF3 or CYLD by somatic alterations also attenuates IRF3 signaling. Furthermore, LTBR amplification and LMP1 overexpression inactivate TRAF3 activity, thereby inhibiting the innate immune responses for EBV infection through impairment of IRF3 signaling and type I interferon production ${ }^{2,3,23}$. In summary, to avoid the EBVinduced innate immune response by attenuating IRF3 signaling, either acquirement of somatic alterations or overexpression of LMP1 were demonstrated in a total of 55/70 (78.6\%) clinical specimens (Fig. 4A).

In terms of adaptive immunity, our data revealed SVs and aberrations of immune checkpoint machinery (PD-L1/CD274; Supplementary figure 5), and antigen presentation molecules including MHC class I ( $H L A-A, H L A-B)$, class II genes ( $H L A-$ DQA1, HLA-DQB2, HLA-DRB1, HLA-DRB5), and their major transcriptional activators (NLRC5 and CIITA) in 22/70 (31.4\%) of NPCs (Fig. 4A). Such alterations could impair antigen presentation by tumor cells allowing them to escape from immune surveillance. In fact, loss-of-function (LOF) alterations in NLRC5 and CIITA were consistent with the loss of MHC class I and class II expression in NPC tumor cells (Fig. 4B and Supplementary figure 6). Notably, inactivating mutations in MHC class I genes (NLRC5, HLA-A,HLA-B) were significantly associated with the presence of APOBEC-related mutational signature (two-sided Fisher's exact test $p=0.002$ ), but not the DNA mismatch repair defect mutational signature. It is likely that the NPCs with high APOBEC3 activity resulting in the presence of the APOBEC mutational signature also acquire inactivating alterations in $\mathrm{MHC}$ class I molecules to enable escape from CD8+ cytotoxic T-cell response. Indeed, a significant relationship between mutational burden and alterations in adaptive immunity-related genes including CD274, NLRC5, HLA-A, HLA-B, CIITA, HLA-DRB1, HLA-DRB5, HLA-DQB2 (two-sided Wilcoxon signed-rank test $p=0.00028)$. These findings suggested that viral factors and somatic events both contribute to defective DNA mismatch repair and APOBEC3 mutational signatures resulting in high TMB, which subsequently favors the selection and clonal expansion of EBV-infected NPC cells bearing somatic alterations in adaptive immunity-related genes.

In addition to somatic events, we also explored potential viralassociated mechanisms for evading host immune responses. Expression of BNLF2a, an EBV lytic gene inhibiting the cellular transporter associated with antigen processing (TAP) protein has been shown to be expressed in gastric cancers latently infected with $\mathrm{EBV}^{26,27}$. From RNA-sequencing data sets of EBVassociated PDXs, we also observed BNLF2a transcription in these NPC models (Fig. 4C, D and Supplementary figure 7). BNLF2a expression was confirmed in the majority of latently infected EBV-positive tumor cells in two NPC PDXs using RNA in situ hybridization (RISH) of a $B N L F 2 a$-specific probe. Because of the overlap of BNLF2a and exon 3 of LMP1 genes, the cases with positive RISH signals for LMP1-specific probe deemed "undetermined". Nevertheless, high levels of BNLF2a transcript alone were detected in at least 13 NPC tumors available for RISH (Fig. 4D).

Notably, a total of $47.1 \%$ (33/70) of NPC tumors had either $B N L F 2 a$ expression or somatic aberrations in antigen presentation and immune checkpoint genes. A trend for mutual exclusivity between high tumor expression of $B N L F 2 a$ and somatic alterations for immune evasion was observed (two-sided Fisher's exact test $p=0.24$ ) (Fig. 4D). These complementary viral 
A

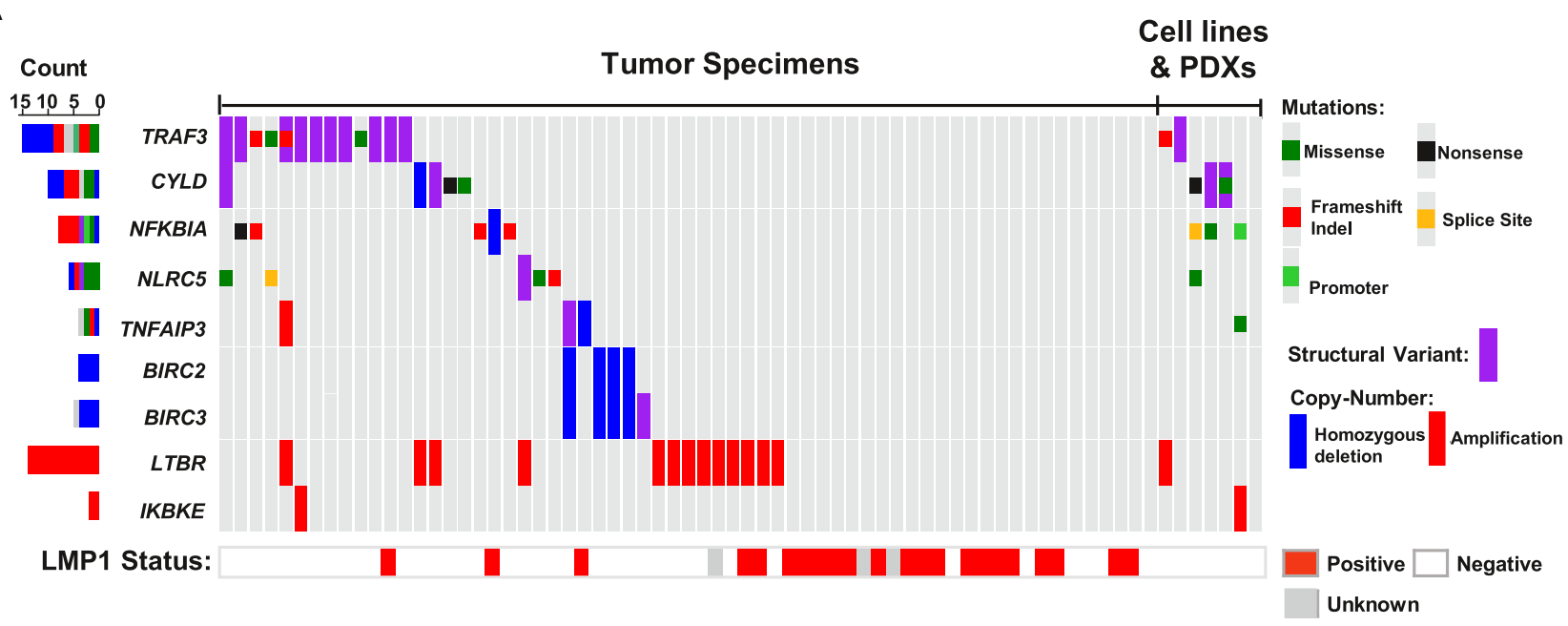

B

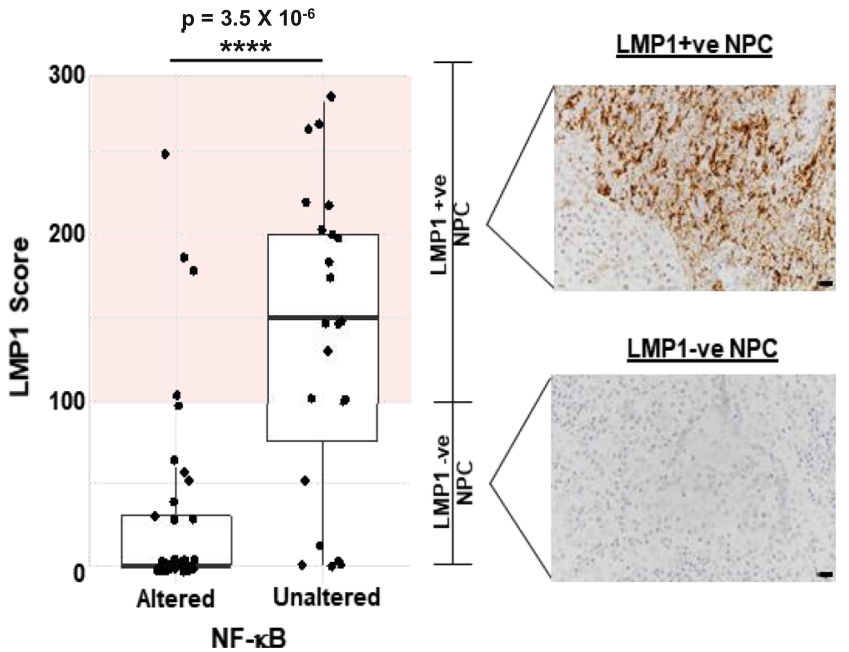

Fig. 3 Aberrant NF-KB pathway activated by somatic gene alterations and EBV latent gene. A Somatic aberrations activating NF- $\kappa B$ and LMP expression in 70 NPC samples. NPC tumors with LMP1 overexpression are also indicated. B LMP1 overexpression and somatic aberrations activating NF-KB pathway are mutually exclusive at the whole-genome level in NPC (two-sided Fisher's exact test $p=3.5 \times 10^{-6},{ }^{\star \star \star \star \star} P<0.0001$ ). Boxplot is defined as follows: center upper whisker $=\min \left(\max (x), Q_{-} 3+1.5{ }^{*} I Q R\right)$, lower whisker $=\max \left(\min (x), Q \_1-1.5 * I Q R\right)$; where IQR = Q_3-Q_1, the bounds of the box. LMP1 expression was examined in NPC tumor specimens $(n=65)$ by immunohistochemistry staining. IHC staining and scoring were performed twice in the tumors and similar results were observed. Representative images of NPC cases with high (+ve) or absence/low ( - ve) LMP1 expression are shown. Scale bar: $10 \mu \mathrm{m}$. Source data are provided as a Source Data file.

and somatic events impairing antigen presentation and immune checkpoints may contribute to the clonal selection for the immune escape of EBV-infected cells during NPC development. This observation of impaired innate and adaptive immunity supports the notion that NPC tumors are protected by both viral and somatic mechanisms of immune evasion in patients, representing the second viral-somatic co-selected mechanisms (after NF- $\mathrm{BB}$ activation) for NPC tumorigenesis.

TGFBR2 loss is a driver event in NPC. Dysregulation of TGF- $\beta$ / SMAD signaling has been shown to initiate cancer formation and disease progression in various human cancers ${ }^{28,29}$. Here our data shows a $24.3 \%$ (17/70 tumors) rate of TGF- $\beta /$ SMAD pathway gene alteration in NPC, targeting TGFBR2, TGFBR3, ACVR2A, and SMAD4 (Fig. 5A). It has been shown that TGFBR2 loss protects EBV-infected NPC cells from autocrine or paracrine TGF- $\beta$-mediated cytostasis and differentiation through Smad signaling ${ }^{28}$. In fact, we found TGFBR2 aberrations in NPC patient tumors, cell lines (NPC43, NPC53) and PDXs (Xeno-32, Xeno38, Xeno-47) (Fig. 5A). Strikingly, using RISH we observed downregulation of TGFBR2 in the majority of clinical tumor samples when compared with adjacent histological normal epithelial cells and infiltrating lymphocytes (Fig. 5B). Similar findings were observed in NPC models (Supplementary figure 8A).

TGFBR2 is a major tumor suppressor residing on chr. $3 \mathrm{p}$, the most frequently deleted chromosomal region in NPC and its precancerous lesions ${ }^{1,2,30}$. To date, however, the role of TGFBR2 loss in the pathogenesis of NPC remains poorly defined. Here, we demonstrated that ectopic TGFBR2 expression inhibited cell proliferation and restored phosphorylation of Smad2/3 in EBVpositive NPC43 cells in response to TGF- $\beta$ stimulation (Fig. $5 \mathrm{C}$ and Supplementary figure $8 \mathrm{~B}$ ). Most importantly, the restoration of TGFBR2 induced differentiation of NPC cells, resulting in marked morphological changes along with the expression of a differentiation marker involucrin (Fig. 5D). In immortalized normal nasopharyngeal epithelial (NPE) cells, NP460, TGFBR2 knockout by CRISPR resulted in inhibition of TGF- $\beta / S M A D$ signaling and 

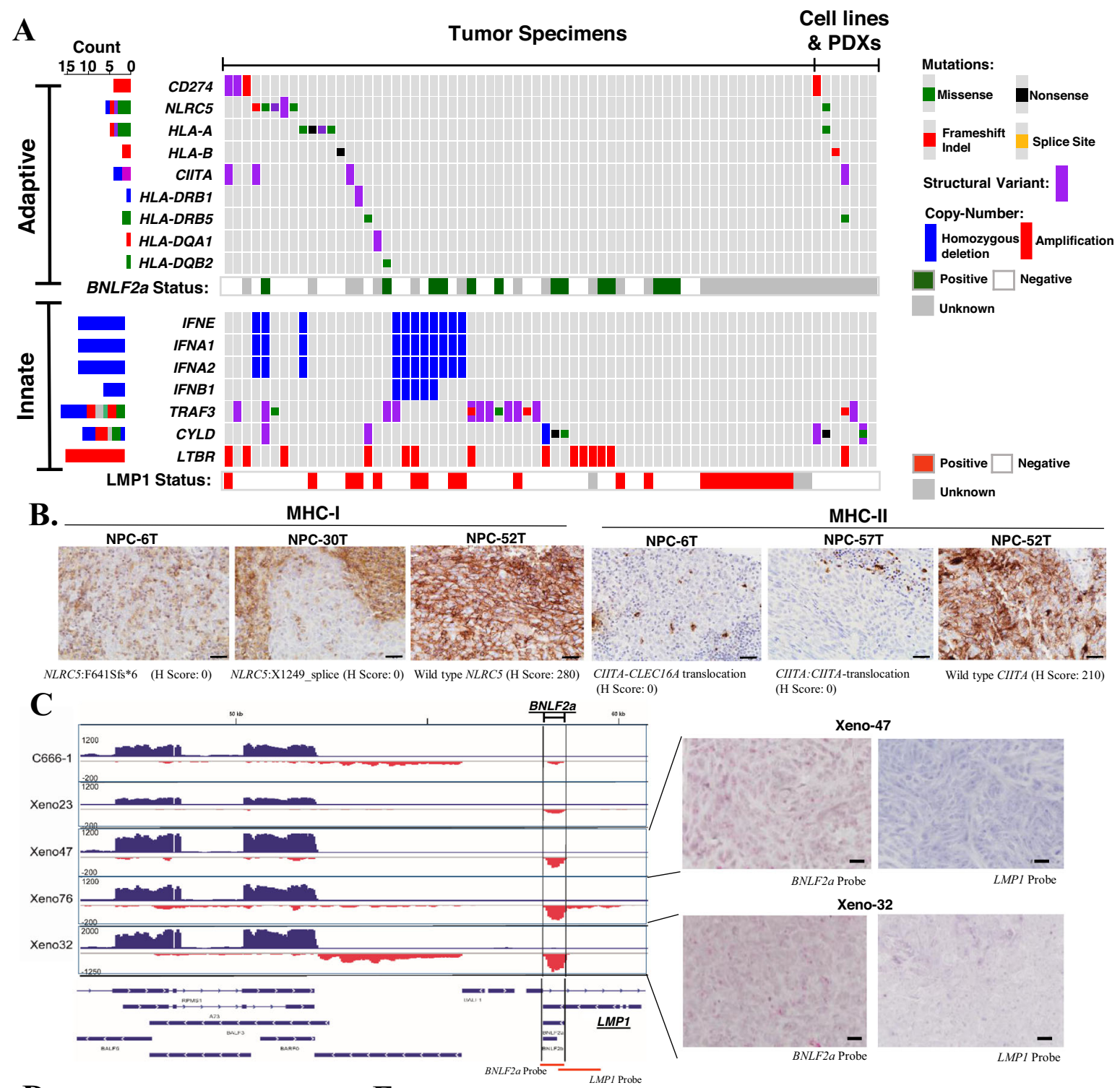

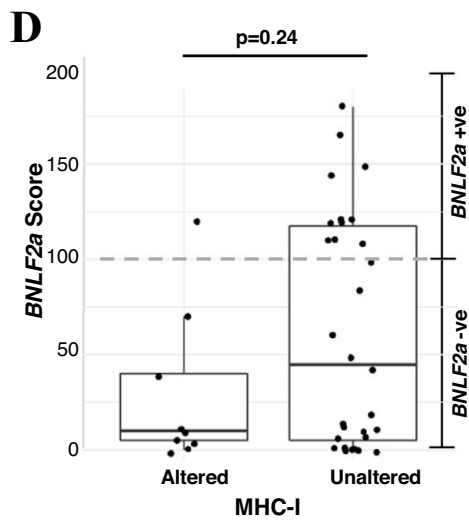

$\mathbf{E}$

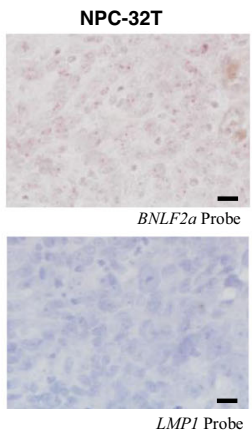

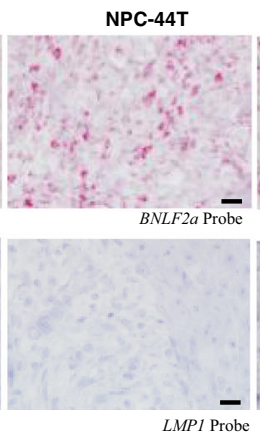
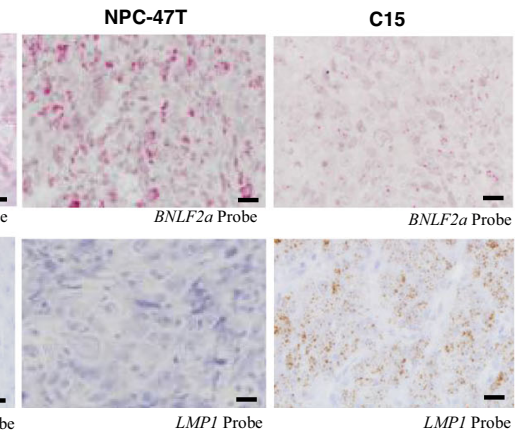

resistance to TGF- $\beta$-induced growth inhibition (Fig. 5E). To examine the effect of disrupting TGF- $\beta /$ SMAD signaling on the outcome and stability of EBV infection in these immortalized NPE cells, the parental NP460 and TGFBR2 knockout NP460KO cells were infected with a green fluorescent protein (GFP)-tagged recombinant EBV (Akata strain), and the EBV-positive cells were isolated using FACS and grown for 7 and 14 days as we described previously ${ }^{31}$. This experiment demonstrated that the efficiency of persistent EBV infection was significantly increased in NPE cells with TGFBR2 inactivation (Fig. 5F and Supplementary figure 8B). Our findings establish TGFBR2 loss as a direct driver for NPC tumorigenesis, playing a pivotal role in attenuated TGF- $\beta$ signaling and establishment of EBV latency.

Genomic aberrations in DNA repair and other oncogenic pathways. While somatic alterations of TP53 and other DNA double-strand break repair genes (e.g., ATM, BARD1, BRCA2, 
Fig. 4 Impaired innate and adaptive immunity by somatic gene alterations and EBV latent gene expression in NPC. A Somatic aberrations and EBVencoded LMP1 and BNLF2a expression attenuate adaptive and innate immunities in NPC are shown. B By immunohistochemistry analysis, loss of MHCclass I and MHC-class II expression are demonstrated in representative NPC cases with somatic alterations of NLRC5 ( $n=3$; NPC-6T, NPC-30T, NPC-52T) and CIITA ( $n=3$; NPC-6T, NPC-52T, NPC-57T), respectively. The experiments were performed twice and similar results were found. Representative images of MHC-class I and MHC-class II expression in the NPC cases with wild-type or somatic alterations of NLRC5 and CIITA genes are illustrated. Scale bar: $20 \mu \mathrm{m}$. C RNA-sequencing revealed the expression of BNLF2a in NPC PDXs. The partial EBV transcription profiles illustrating BNLF2a expression in NPC cell line (C666-1) and PDXs (Xeno-23, Xeno-47, Xeno-76, and Xeno-32) were shown. The RISH probe targeted regions (BNLF2a, LMP1) in EBV genome are indicated. By RISH analysis, abundant expression of BNLF2a transcripts was found in the majority of tumor cells in two NPC PDXs, Xeno-32 and Xeno-47. BNLF2a probe was used to illustrate the signal of BNLF2a transcripts in the tumor cells with no LMP1 probe signal. Each tumor was subjected to RISH analysis twice and similar results were found. Representative images of RISH results of BNLF2a and LMP1 in NPCs are illustrated. Scale bar: $10 \mu \mathrm{m}$. D Enrichment of BNLF2A-expressing cases in NPCs without somatic alterations in MHC-class I pathway (two-sided Wilcoxon signed-rank $p=0.24$ ). Boxplot is defined as follows: center upper whisker $=\min \left(\max (x), Q \_3+1.5^{\star} \mid \mathrm{QR}\right)$, lower whisker $=\max \left(\min (\mathrm{x}), \mathrm{Q}_{-} 1-1.5^{\star} I Q R\right)$; where IQR $=$ Q_3-Q_1, the bounds of the box. BNLF2A expression was determined in NPC specimens $(n=39)$ by RISH. E Representative NPC tumors (NPC-32T, NPC-44T, NPC-47T) with high BNLF2a expression are shown. An LMP1-expressing xenograft C15 was included for RISH analysis using BNLF2a and LMP1 probes. Each tumor was subjected to RISH analysis twice and similar results were found. Scale bar: $10 \mu \mathrm{m}$. Source data are provided as a Source Data file.

CHECK2, RAD51B) were identified in 20/70 (28.6\%) of NPC, we noticed that the HR signature positive tumors were significantly in possession of these mutations (two-sided Fisher's exact test $p=$ 0.044; Figs. 1A and 6). Notably, HR-defect- signature positivity was associated with a significant increase in SV count (two-sided Wilcoxon signed-rank test $p=0.016$; Supplementary figure 9A). Extensive genome-wide structural alterations (SV count $>150$ ) were observed in two NPC clinical specimens (NPC-24T and NPC-38T; Supplementary figure 9A). Both of these highly rearranged tumors had deleterious alterations in an $\mathrm{HR}$ pathway member. Specifically, NPC-24T harbored an ATM-NCOR1 fusion leading to truncation of $A T M$ at exon 36, whereas NPC-38T displayed a focal region on chr13q with numerous breakpoints, characteristic of chromothripsis, leading to homozygous loss of BRCA2(Supplementary figure 10). The most commonly altered DNA repair gene TP53, was associated with a significant reduction in disease-free and overall survival (Supplementary figure 9B). However, patients with mutations in other HR-related gene defects did not experience a different outcome, indicating the TP53-altered status is likely associated with disease aggressiveness through mechanisms other than genomic instability, such as cell cycle regulation.

We also observed alterations to the cell cycle machinery alterations affecting $74.3 \%(52 / 70)$ of NPC cases at the wholegenome level. Aside from the aberrations of TP53, homozygous deletion and structural alterations of CDKN2A ranked at the top (42.9\%; 30/70 cases), followed by amplification of CCND1, CCND2, CDK4, and CDK6 (31.4\%; 22/70) (Fig. 6, Supplementary figure 11). This is consistent with previously reported roles of the $C D K N 2 A / C C N D 1 / C D K 4$ axis in NPC cell growth and persistent EBV latent infection in NPE cells ${ }^{1,2,31}$.

Frequent genomic events were also found within the NOTCH signaling pathway $(14 / 70 ; 20 \%)$ along with members of those affecting the chromatin modification machinery $(29 / 70 ; 41.4 \%)$ (Fig. 6, Supplementary figure 10). In the NOTCH pathway, relatively common alterations in $\mathrm{NOTCH} 1(5 / 70 ; 7.1 \%)$, and MAML2 (5/70; 7.1\%) were noted (Supplementary figure 12). Identification of LOF events, such as inactivating gene rearrangements of NOTCH1 and MAML2 support their tumor-suppressive roles in NPC, similar to that described in other head and neck cancers (Supplementary figure 13) ${ }^{32}$. Multiple recurrent aberrations impairing chromatin modification machinery included ARID1A (5.7\%), ARID1B (10\%), CHD6 (4.2\%), KMT2C (5.7\%), KMT2D (11.4\%), KDMA6A (8.6\%), and EP300 (10\%) (Fig. 6, Supplementary figure 12). Interestingly, LOF alterations were also commonly found in a cluster on chr 3 p21.3 region encompassing genes of the SWI/SNF complex (BAP1 and PBRM1) and chromatin remodeling (SETD2 and BAP1) (11/70, 15.7\%) (Supplementary figure 12).

MTAP deletion is druggable in primary and recurrent NPC. Previous exome studies have shown that there are many potentially druggable targets scattered across the NPC genome ${ }^{2,8-11,13,14}$. Our whole-genome study also revealed hotspot aberrations of common drug targets including PIK3CA, $E G F R, B R A F, M E T$, and $E R B B 2$, yet these appeared infrequently (totaling 2.9\%, 2/70). Druggable fusion events were identified in $7.1 \%(5 / 70)$ of tumor specimens: FGFR3-TACC3 $(n=2)$, NTRK2 fusions $(n=1), \operatorname{ALK}(n=1)$, and ROS1 $(n=1)$. In the case of NTRK and ROS1 fusions, the FDA-approved pan-cancer drugs, larotrectinib, and entrectinib are readily available for clinical treatment in these subsets of NPC patients. Recently in classic Hodgkin lymphoma, a 9p24.1 amplification involving CD274 $(P D-L 1)$ and PDCD1LG2 (PD-L2) was shown to predict response to nivolumab outcomes ${ }^{33}$. As a target for immunotherapy, $P D-L 1$ displayed structural alterations in only 5.7\% (4/61) of NPC cases (Fig. 4). Amidst the positive clinical trial results of anti-PD-1 immunotherapy in NPC with $20-34 \%$ overall response rates, it would be important to determine if $P D-L 1$ genomic aberrations can predict outcomes for $\mathrm{PD}-1 / \mathrm{PD}-\mathrm{L} 1$ targeting in clinical settings ${ }^{34-36}$. In a recent phase II clinical study of PD-1 inhibitor for recurrent and metastatic NPC, Wang et al. identified a potential association of genomic amplification in $11 \mathrm{q} 13$ regions and ETV6 alterations with poor response ${ }^{14}$. Thus, comprehensive evaluation of both viral factors and somatic alterations targeting host immunity in these clinical trials may help to identify patients who may respond to immunotherapies.

Strikingly, an emerging drug target, MTAP (methylthioadenosine phosphorylase) was identified as a commonly deleted gene by WGS, accounting for $34 \%$ (24/70) of NPC cases. MTAP is a key enzyme for polyamine metabolism and salvaging of adenine and methionine. Studies have revealed the pharmacologic vulnerability of MTAP-deficient tumors through targeting the MAT2A/ PRMT5 axis ${ }^{37-40}$. MTAP resides next to CDKN2A on chr. 9p21.3 and is often co-deleted with $C D K N 2 A$, a frequently deleted tumor suppressor in human cancers. In fact, homozygous deletions of MTAP and CDKN2A are almost in complete overlap in our NPC cohort and several PDXs including xeno-76 (Fig. 2). In an independent cohort of recurrent NPC, FISH analysis concluded that $32 \%(16 / 50)$ of aggressive tumors harbored MTAP homozygous deletion (Fig. 7A, supplementary data 6). The loss of MTAP expression was confirmed in these cases by IHC staining (Fig. 7B and supplementary figure 14A). In addition, the co- 

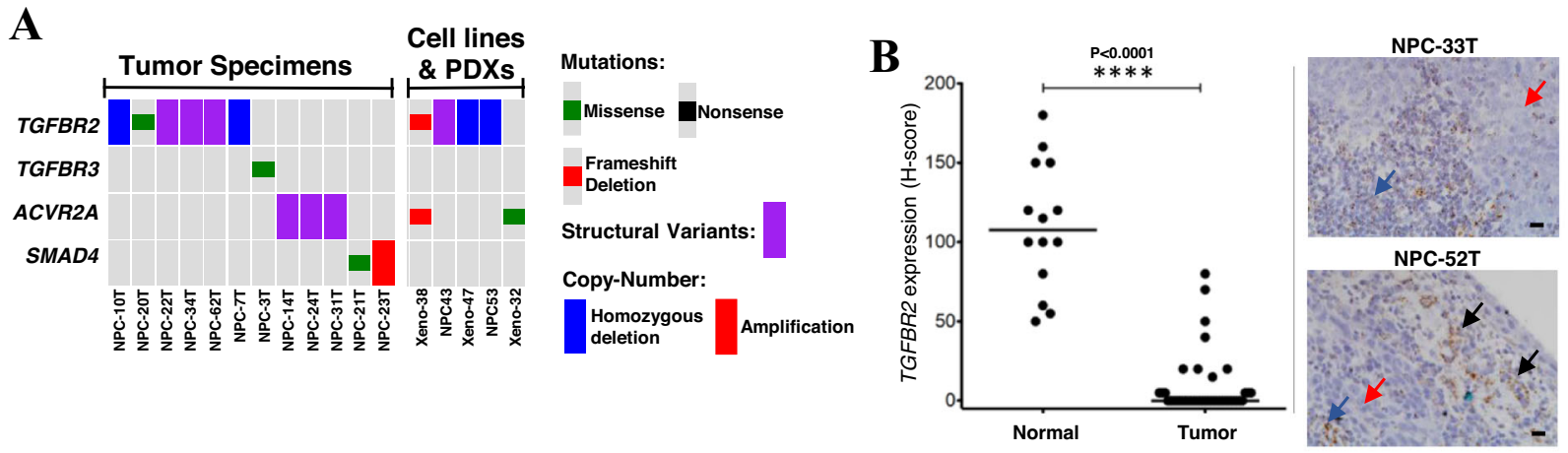

C

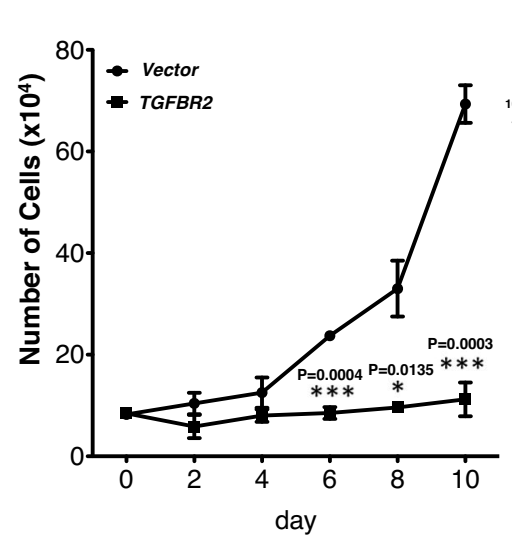

$\mathbf{E}$

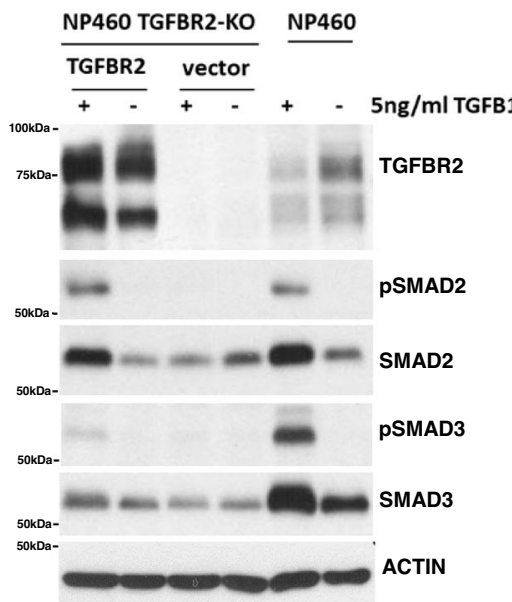

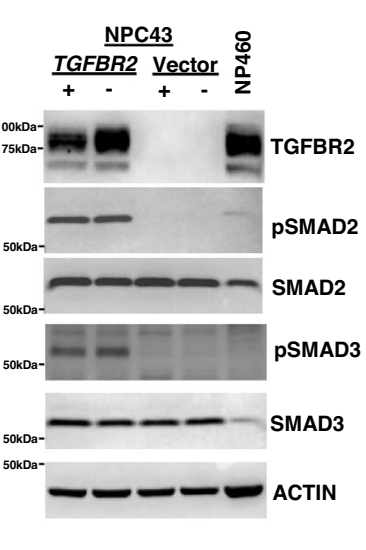

D

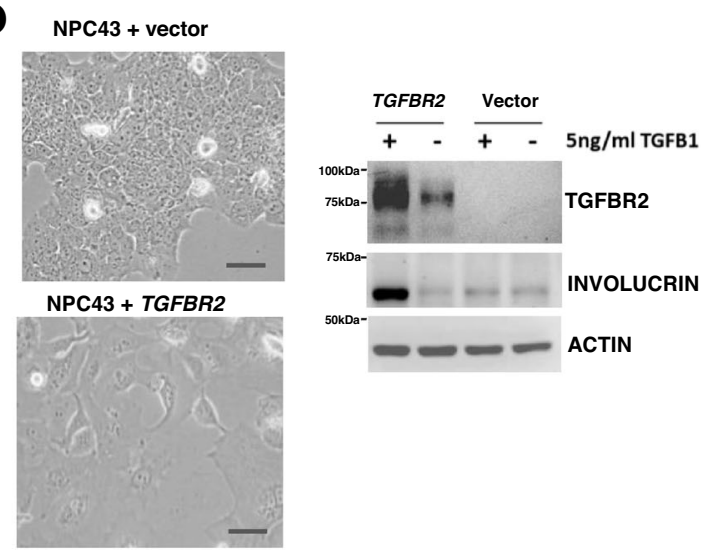

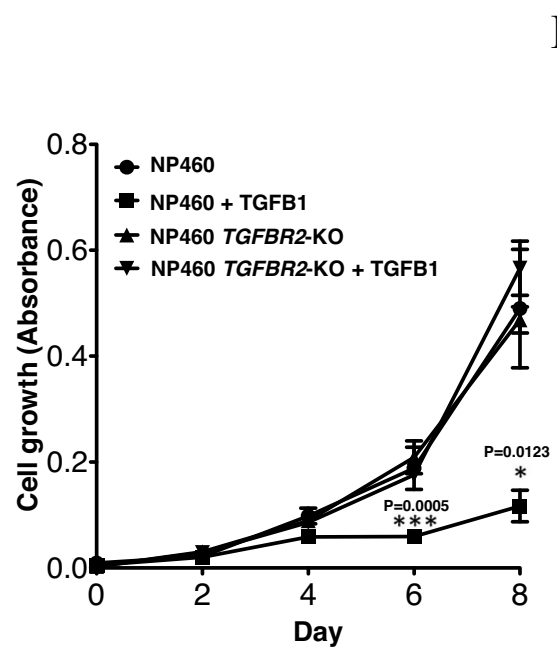

$\mathbf{F}$
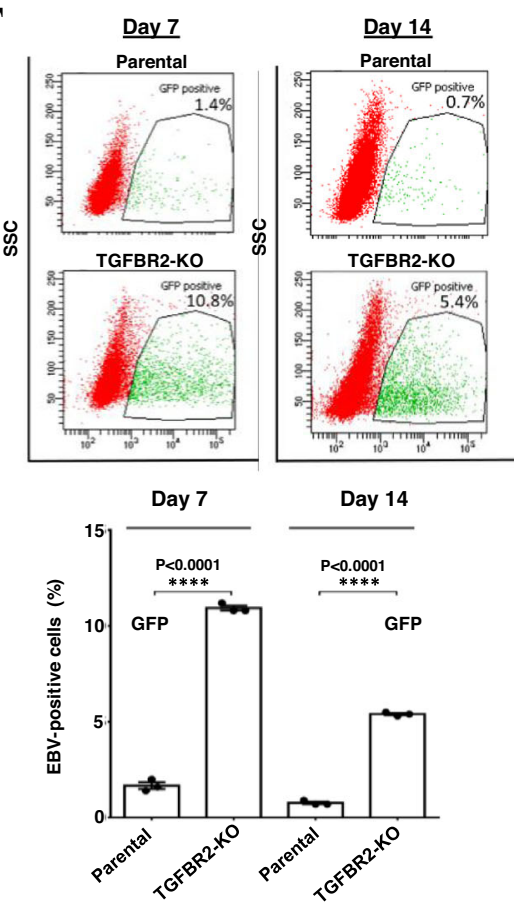

deletion of MTAP and CDKN2A were also confirmed in 7-16.7\% of NPC samples in previous whole-exome and WGS studies (Supplementary figure 14B) $)^{12,14}$.

We further examined the responsiveness of MTAP-deficient NPC to MAT2A inhibition in vitro and in vivo. MTAP-null C666-1 cells were generated by CRISPR and subjected to treatment with MAT2A siRNA, PRMT5 siRNA and chemical inhibitor treatments (Fig. 7C-F). As shown in Fig. 7C, the level of symmetric dimethylarginine (SDMA) was markedly reduced in MTAP-null C666-1 cells in response to MAT2A and PRMT5 siRNA treatments, indicating significant inhibition of PRMT5 activity. Knockdown of MAT2A and PRMT5 significantly suppressed the growth of MTAP-null C666-1, but not that of the parental MTAP-WT C666-1 cells (Fig. 7D). Importantly, MTAP-null C666-1 cells demonstrated heightened sensitivity ( $\sim 8.7$-fold more sensitive) to the MAT2A inhibitor, FIDAS-5, 
Fig. 5 TGFBR2 loss as a driver event in NPC. A Somatic alterations of TGFBR2 and other genes in TGF- $\beta$ /SMAD pathway. B RISH analysis of TGFBR2 expression in 41 NPCs and 14 adjacent normal epithelium. Significant downregulation of TGFBR2 was observed (Unpaired two-tailed $t$ test, ${ }^{\star \star \star \star} p<0.0001$, mean values of the data are presented) in NPC. Representative NPC cases (NPC33T, NPC-52T) showing no TGFBR2 expression in the tumor cells (red arrows) and positive signals in the infiltrating lymphocytes (blue arrows) and normal epithelium (black arrows). Scale bar: $10 \mu \mathrm{m}$. C Dramatic growth inhibition was found in wild-type TGFBR2 transfected TGFBR2-deleted NPC43 cells (unpaired two-tailed $t$ test, ${ }^{\star} p<0.05$; ${ }^{\star \star \star} p<0.0005$; data are presented as mean values \pm SEM). Western blotting showed pSMAD2 and pSMAD3 overexpression in TGFBR2-expressing NPC43 cells. NP460 with TGFBR2 expression was included as control. Replication: $n=3$ biologically independent experiments. D Re-introduction of wild-type TGFBR2 induced cell differentiation and morphological changes in NPC43 cells. In TGFBR2-expressing NPC43 cells, the levels of involucrin were increased upon TGF- $\beta 1$ treatment, as compared with the vector control. Replication: $n=3$ biologically independent experiments. Similar results were found in the repeated experiments. Scale bar: $50 \mu \mathrm{m}$. E CRISPR/Cas9-mediated knockout of TGFBR2 in NP460 (NP460KO) cells resulted in the loss of TGFBR2. No pSmad2 and pSmad3 expression were detected in NP460KO cells following TGF- $\beta 1$ treatment. Re-expression of wild-type TGFBR2 in NP460KO cells restored its response to TGF- $\beta 1$ as shown by the induction of $\mathrm{pSmad} 2$ and $\mathrm{pSmad3}$. NP460KO cells showed significant growth inhibition when compared with parental NP460 cells after TGF- $\beta 1$ treatment (unpaired two-tailed $t$ test, ${ }^{\star} p<0.05$; ${ }^{\star \star \star} p<0.0005$; data are presented as mean values \pm SEM). Replication: $n=5$ biologically independent experiments. F The parental NP460 and NP460KO cells were infected with a GFP-tagged recombinant EBV. After EBV infection and flow sorting of EBV-positive cells, significantly higher numbers of EBV-positive cells were maintained in TGFBR2 knockout NP460KO cells than those in parental cells on both day 7 and day 14 (unpaired two-tailed $t$ test, ${ }^{\star \star \star \star} p<0.0005$, data are presented as mean values \pm SEM). Replication: $n=3$ biologically independent experiments. Source data are provided as a Source Data file.

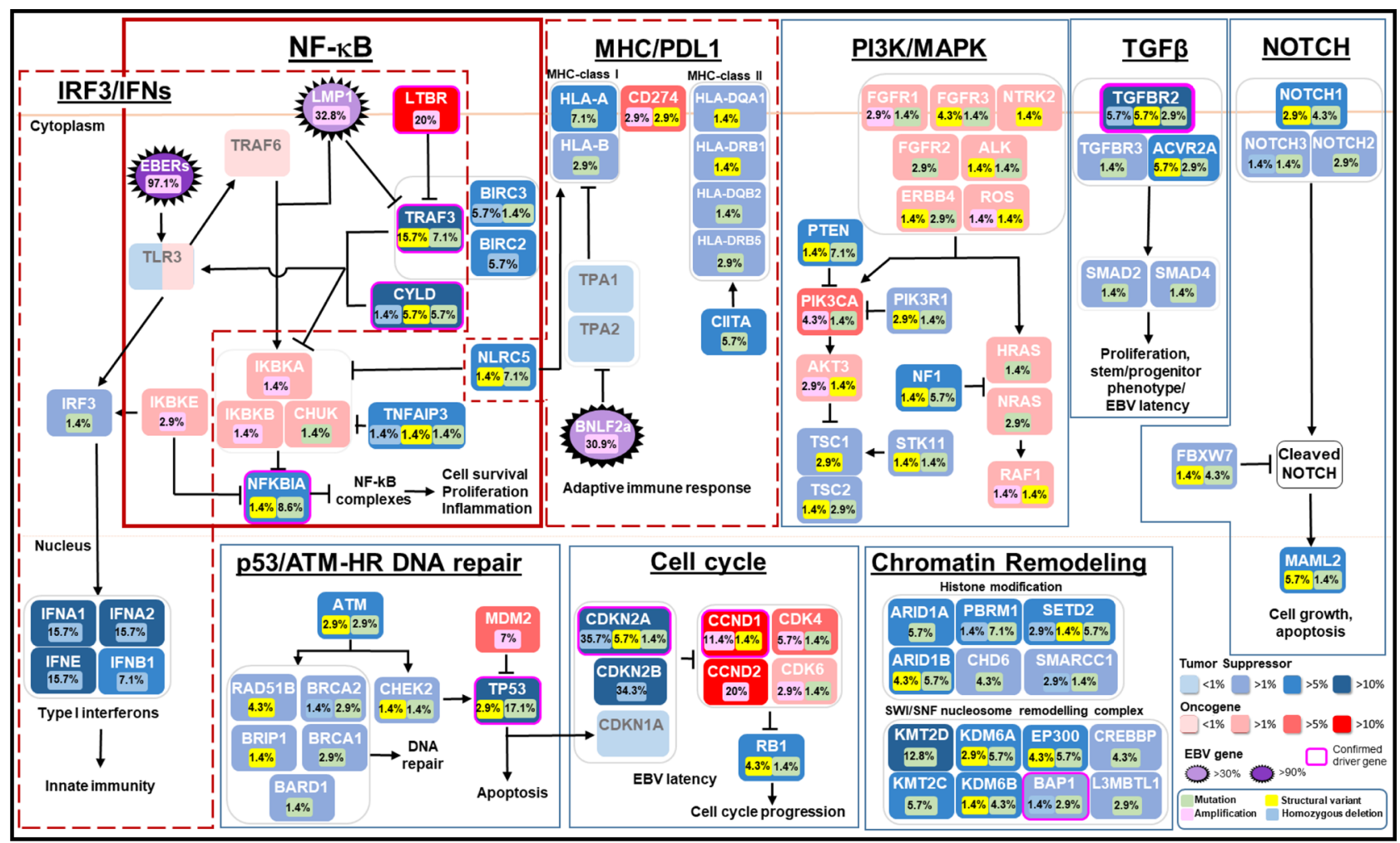

Fig. 6 Frequently altered cancer pathways in NPC at the whole-genome level. Multiple genomic aberrations and EBV latent genes alter multiple cancer pathways in NPC.

compared with MTAP-wild-type NPC cell lines (Fig. 7E). FIDAS5 treatment resulted in a reduction in the level of SDMA, accompanied by p53 expression in MTAP-null C666-1 cells (Fig. 7F). In vivo, FIDAS-5 elicited marked antitumor activity in an MTAP-deficient NPC PDX, Xeno-76 while no such antitumor effect was observed for MTAP-WT C666-1 tumors (Fig. 7G, H). In Xeno-76-transplanted mice treated with FIDAS-5, the level of circulating EBV DNA was significantly reduced, associated with an obvious reduction in tumor burden (Fig. 7I). Marked suppression of SDMA was accompanied by the increase of cleaved caspase 3, p53, BAX, and involucrin in the MTAPdeficient Xeno-76 tumors (Fig. 7J). At last, FIDAS-5 treatment resulted in the occurrence of tumor cells with keratinization phenotype and involucrin expression in Xeno-76, but not in
MTAP-WT C666-1 tumors (Fig. 7H and Supplementary figure $14 \mathrm{C}$ ). Here, we documented the therapeutic vulnerability of MTAP-deficient NPC by a MAT2A inhibitor. This precision strategy warrants future assessments in clinical studies, which might well impact a large subset of NPC patients.

\section{Discussion}

The present WGS study establishes a comprehensive wholegenome landscape of a large NPC cohort $(n=70)$, which can serve as a useful resource for the broader community. In addition to genome-wide mutations, rearrangements, and accurate copy number $(\mathrm{CN})$ changes our study incorporates EBV viral gene expression, revealing multiple important insights into NPC 


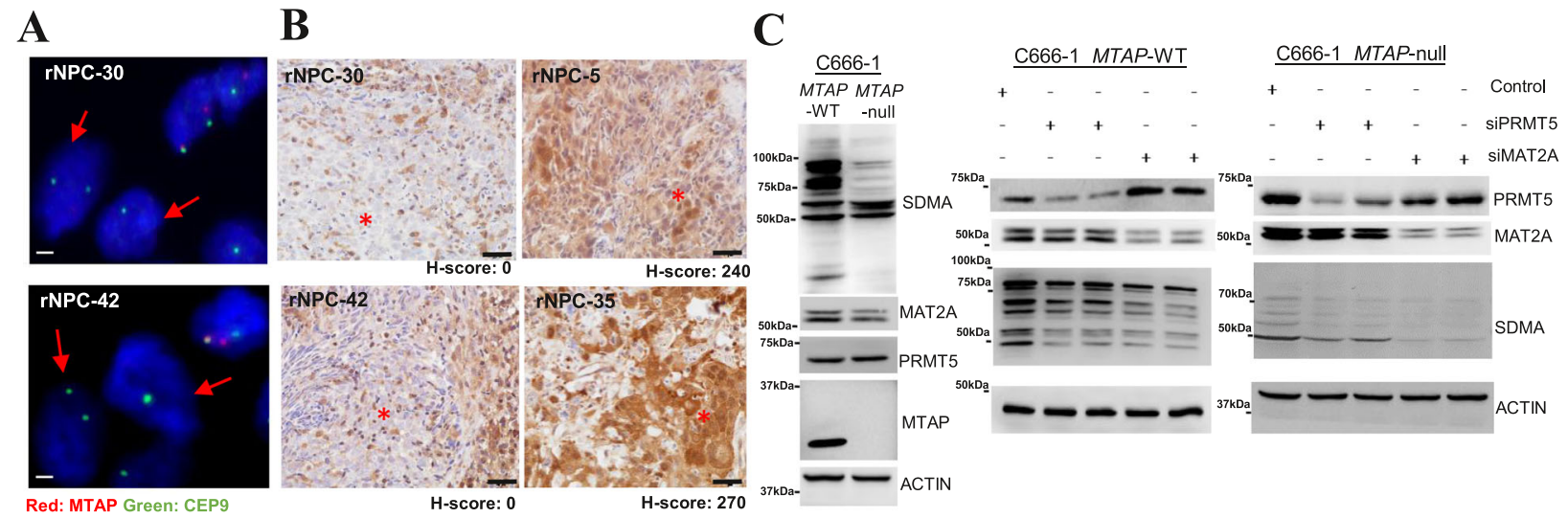

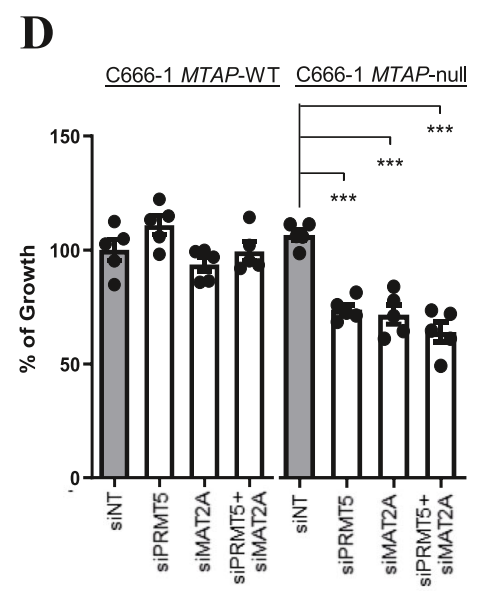

G

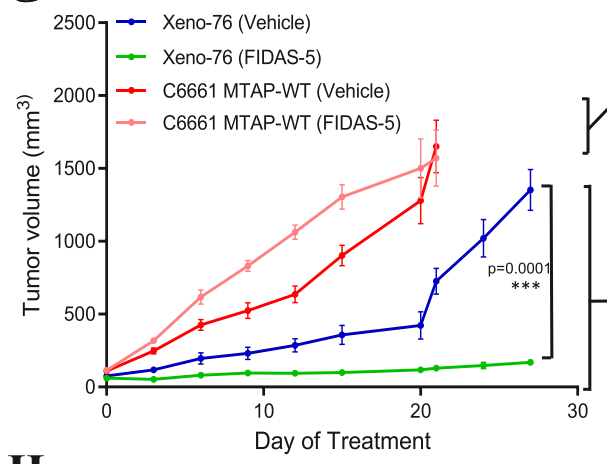

$\mathbf{H}$

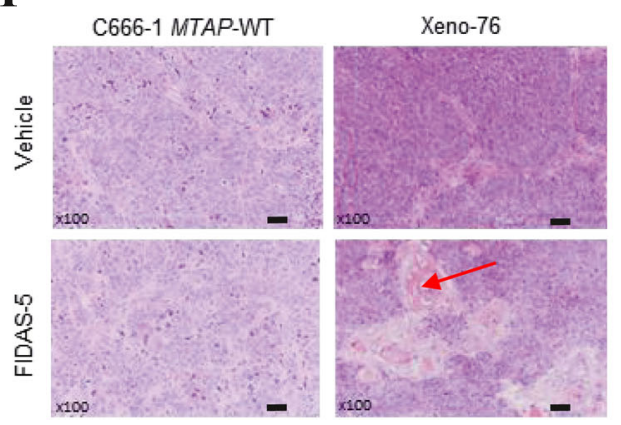

fouss

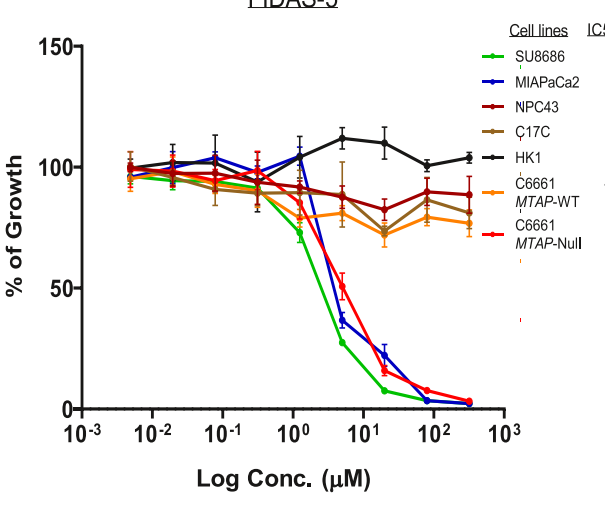

$\mathbf{J}$
F

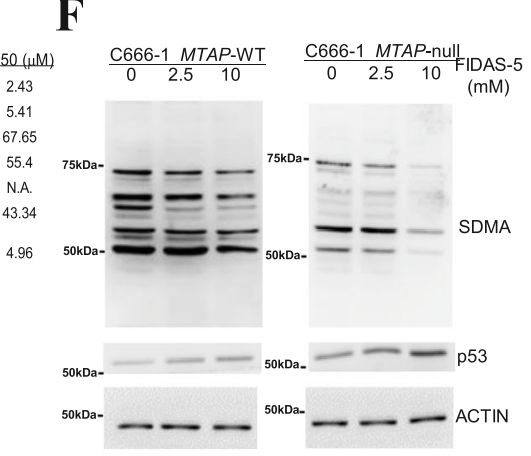

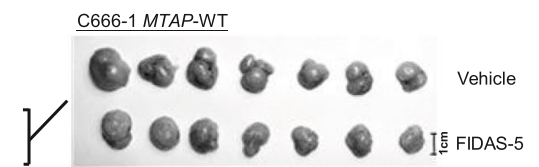
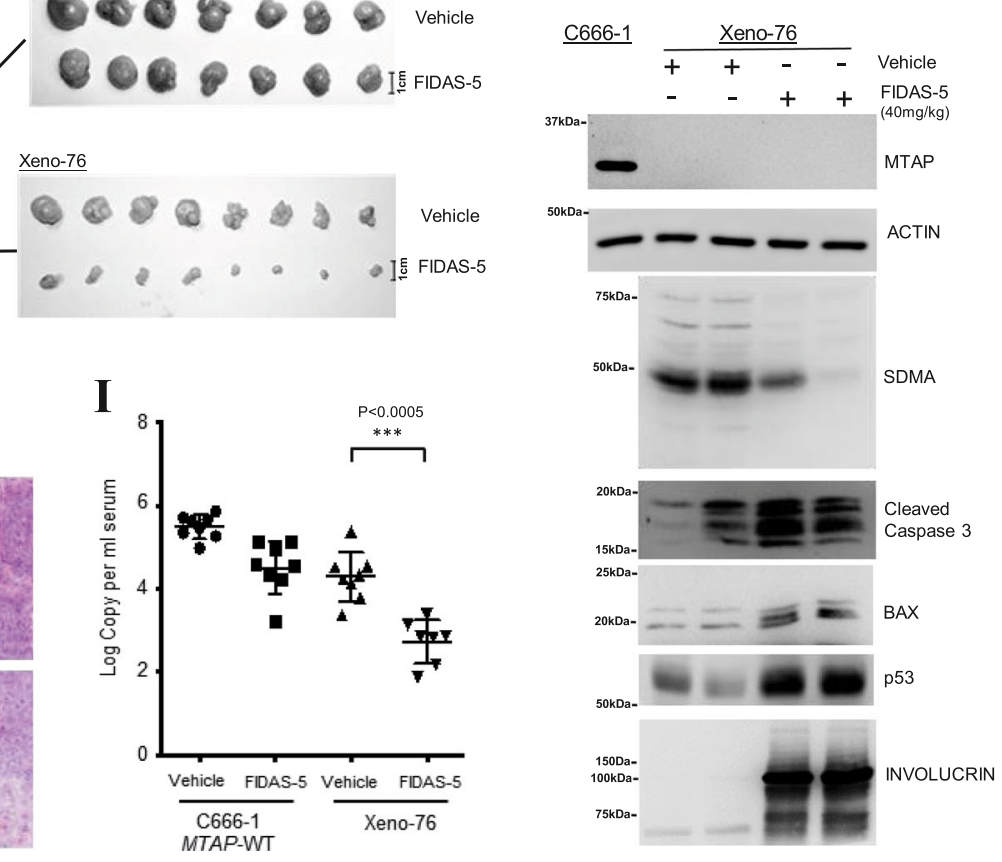

tumorigenesis further establishing the interrelated roles for both host somatic alterations and EBV gene expression impacting multiple cellular mechanisms, namely NF- $\kappa B$ activation, immune evasion, and persistent infection with $\mathrm{EBV}^{8-11,13,14}$.

Firstly, our results show that constitutive activation of the NF- $\kappa B$ inflammatory pathways occurs in as high as $90 \%$ of NPC either through somatic alterations or expression of the virus-encoded LMP1 oncogene, implicating aberrant NF- $\kappa \mathrm{B}$ activation as a ubiquitous hallmark of this EBV-associated malignancy. The dominance of NF- $\kappa B$ activation in NPC that we have identified is consistent with recent genome-wide CRISPR-based gene knockout screens and functional studies showing that perturbation of the NF- 
Fig. 7 MTAP deletion is pharmacologically vulnerable to MAT2A inhibition. A FISH analysis detected homozygous deletion of MTAP in two NPC tumors (rNPC-30, rNPC-42). Scale bar: $1 \mu \mathrm{m}$. B Loss of MTAP expression was confirmed in MTAP-deleted NPC tumors (rNPC-30, rNPC-42) by IHC. In two NPC tumors without MTAP deletion, rNPC-5 and rNPC-35, representative images of MTAP expression were shown. Scale bar: $20 \mu \mathrm{m}$. Similar results were found in $n=2$ independent FISH and IHC experiments. C Loss of MTAP expression and reduced SDMA in MTAP-null C666-1 cells. Knockdown of MAT2A and PRMT5 by siRNAs repressed SDMA in MTAP-null C666-1 cells. Similar effects were observed in $n=3$ biologically independent experiments. D Knockdown of MAT2A and PRMT5 significantly inhibited cell growth of MTAP-null C666-1 cells. (unpaired two-tailed $t$ test, ${ }^{* \star \star} p<0.0005$; data: mean values \pm SEM; $n$ $=5$ biologically independent experiments). E FIDAS- 5 inhibited the cell growth in MTAP-null C666-1 cells, but not parental C666-1 and MTAP-intact NPC cells (NPC43, C17C, HK1). The MTAP-deleted SU8686 and MIAPaCa2 cells were included as controls. IC50 of each cell line is shown (Data: mean values \pm SEM). Replication: $n=5$ biologically independent experiments. F Reduced SDMA and increased p53 expression were observed in FIDAS-5 treated MTAPnull C666-1. Similar findings were detected in $n=3$ biologically independent experiments. G Marked in vivo inhibition of MTAP-deleted Xeno-76 by FIDAS5. Growth curves and harvested tumors of C666-1 (MTAP-WT) $(n=8)$ and Xeno-76 $(n=7)$ in nude mice treated with vehicle or FIDAS-5 are shown (unpaired ANOVA test, ${ }^{\star \star \star} p=0.0001$, data: mean values \pm SEM). H The morphological changes (cell differentiation and keratin production (red arrow)) are illustrated in representative H\&E-stained tissue sections of FIDAS-5 treated Xeno-76. Scale bar: $50 \mu \mathrm{m}$. I Circulating EBV DNA copies were significantly reduced in the Xeno-76 mice after FIDAS- 5 treatment. (Unpaired two-tailed $t$ test, ${ }^{\star \star \star} p<0.0005$; data: mean values \pm SEM). The blood samples examined in each treatment group of Xeno-76 and C666-1 MTAP-WT are $n=8$ and $n=7$, respectively. J FIDAS-5 treated Xeno-76 showed reduced SDMA, and increased cleaved caspase 3, BAX, p53, and involucrin expression. Similar effects were shown in $n=3$ biologically independent experiments. Source data are provided as a Source Data file.

$\kappa \mathrm{B}$ pathway significantly inhibited the growth of EBV-positive NPC cells ${ }^{8,20,41,42}$. These studies, in conjunction with our current findings, conclude the essential role of constitutively activated NF- $\mathrm{BB}$ signaling in NPC, a feature distinct from the heterogeneous genomic landscapes of other head and neck cancers.

Second, we identified the mutually exclusive involvement of somatic alterations and overexpression of various viral genes $(L M P 1, B N L F 2 a)$ targeting innate and adaptive immunity in 91.4\% of NPCs. This viral and somatic co-selection is the second viral-somatic collaboration we identified herein. This feature likely arises to counteract the inflammatory environment owing to persistent EBV infection, a unique feature of NPC. Under normal circumstances, the abundantly expressed viral RNAs (e.g., EBERs) would trigger potent innate immune response via IRF3activated type I interferon production while the presentation of multiple immunogenic viral antigens (e.g., EBNA1, LMP2) would facilitate the cytotoxic T-cell responses in EBV-infected $\mathrm{NPC}^{2-4,24,25}$. Thus, effective evasion from immune responses is believed to play a key role in the tumorigenesis of this virally associated cancer. In essence, our study defined a panel of genomic and viral events interfering with innate and adaptive immune responses in $78.6 \%$ and $47.1 \%$ of tumors, respectively, uncovering major mechanisms for NPC immune evasion. Notably, we confirmed the overexpression of the viral TAP inhibitor $B N L F 2 a$ in the latent EBV-infected cells of $18.6 \%$ of NPC tumors, which could counteract host immune response. The identification of $B N L F 2 a$ expression, PDL1 alterations, and other somatic alterations impairing the antigen presentation machinery may inform immunotherapy-related biomarkers or strategies in NPC.

Third, WGS revealed consistent loss of expression and frequent somatic LOF alterations of CDKN2A and TGFBR2, corroborating their key driver roles in NPC development. In addition to its known role in suppressing cell proliferation and differentiation, our study provides evidence supporting a pivotal role for TGFBR2 inactivation in persistent EBV infection in NPC. Disruption of TGF- $\beta$ signaling in NPE cells may facilitate the maintenance of the EBV genome via suppression of cellular differentiation ${ }^{29}$. Since deletion of chr. $3 \mathrm{p}$ and $9 \mathrm{p}$ which harbor the TGFBR2 and $C D K N 2 A$ loci, respectively, are consistently found in precancerous lesions of NPC, pointing to a central role for CDKN2A loss and impaired TGF- $\beta$ signaling in creating a susceptible NPE cell population capable of supporting EBV latency during early cancer development ${ }^{1,2,29-31}$.

Finally, a combination of genomics and therapeutic investigation demonstrated the pharmacologic vulnerability of MTAP-deleted NPC, which accounts for $32-34 \%$ of our cohort. MTAP is also commonly deleted in glioblastoma (40\%), melanoma (25\%), and pancreatic adenocarcinoma $(25 \%) ;^{37-40}$ unlike NPC however, those cancers are commonly TP53-mutated. Since the majority of MTAPdeleted NPC harbors wild-type p53, the high levels of p53 induction triggered by MAT2A inhibitor may underlie the potent tumor suppressor activity observed in NPC in contrast to other MTAPdeleted but largely TP53-mutated cancers. At present, a phase I clinical trial of MAT2A inhibitor AG-270 (NCT03435250) is ongoing for patients with advanced or refractory solid tumors and lymphomas. We believe our findings will encourage future clinical trials examining MAT2A inhibitors in MTAP-deleted NPC, including recurrent and metastatic diseases, which are often deadly. This precision medicine strategy, together with potential biomarkerdriven immunotherapy may ultimately improve treatment outcomes for patients with advanced NPC.

In conclusion, we have established a comprehensive genome landscape of NPC, and greatly enriched our understanding of NPC etiologies and driver events for tumorigenesis. We demonstrate further the interplays between EBV and somatic alterations, which are co-selected during NPC evolution. Most importantly, our findings provide a rational basis for future precision therapy trials potentially impacting $>30 \%$ of primary, advanced and recurrent NPC, for the classes of MAT2A and PRMT5 inhibitors.

\section{Methods}

NPC cell lines, PDXs, and patient tumor samples. All 63 NPC tumor tissues (including two specimens from the same donor) were collected by endoscopy or surgery, embedded in optimal cutting temperature compound, and stored at $-70{ }^{\circ} \mathrm{C}$ DNA samples extracted from microdissected frozen tissue sections were subjected to WGS. For all patients, corresponding blood samples were collected as normal control. Written patient consents were obtained from all patients in this study according to institutional clinical research approval. The study protocol was approved by the Joint Chinese University of Hong Kong-New Territories East Cluster Clinical Research Ethics Committee at the Chinese University of Hong Kong, Hong Kong SAR. EBV infection status in these tumor samples was confirmed by EBER in situ hybridization ${ }^{12}$. The detailed information of the tumors was provided in supplementary data 1 . Three NPC cell lines (NPC43, NPC38, NPC53) and four patient-derived xenografts (xeno-23, xeno-32, xeno-47, xeno-76) recently established by us were included for WGS ${ }^{12}$. Among these samples, 15 tumor specimens, 3 cell lines, and 4 xenografts were also included in our previous exome and genome sequencing studies 8,15 . For functional and preclinical studies, the immortalized NPE cells (NP69, NP460) and authenticated EBV-positive cell lines (C666-1, C17C) and PDXs (xeno-2117, C15, C17) established by us and Prof. Pierre Busson were also used $41,43,44$. The establishment of MTAP-deleted C666-1 and TGFBR2 knockout NP460 cells is described in supplementary methods. All NPC and immortalized NPE cell lines used in this studies cell lines were established by our team (KW Lo and SW Tsao) and have been authenticated by STR (short tandem repeat) profiling and EBER in situ hybridization ${ }^{41,43,44}$. The cell lines were tested to be negative for mycoplasma contamination by PCR analysis using primer $5^{\prime}$-YGCCTGVGTAGTA YRYWCGC-3' (MYCO5) and 5'-GCGGTGTGTACAARMCCCGA-3' (MYCO3). No commonly misidentified cell lines listed in ICLAC were used in this study. 
Whole-genome sequencing. For tumor and normal samples, $1 \mu \mathrm{g}$ of genomic DNA was subjected to WGS with a standard 100-bp paired-end read using the Illumina HiSeq 2000 platform (Illumina, San Diego) based on the manufacturer's instructions ${ }^{8}$. Sequencing libraries were constructed with 500 bp insert length. Raw sequence reads were processed and aligned to the hg38 human reference genome using bwa-mem (v0.7.15). Local realignment was performed around indels using GATK (v3.6-0) and duplicates were marked using Picard (v2.6.0). Mean target coverage of $51 \times$ and $83 \times$ was achieved for the normal and tumor samples, respectively. BAM files are deposited to the European Genome-phenome Archive under the accession EGAS00001004705.

Variant calling. Somatic SNVs and small insertions and deletions (indels) were detected using four callers and several downstream filters (see Supplementary Methods). Significantly mutated coding genes and non-coding regulatory elements were identified using ActiveDriverWGS (v0.0.1). Coding genes with mutation rates above background were filtered to include those with more than three nonsynonymous mutations excluding notorious passengers ${ }^{19}$. Non-coding calls were limited to open chromatin regions (by ATAC-seq in defined regulatory elements; detailed in Supplementary Methods). SVs were called using three callers and merged, validated, and annotated using MAVIS (v1.8.5), and refined using several downstream filters (see Supplementary Methods).

CN calling. Allele-specific CN profiles were generated using Varscan2 (v2.3.6) and the R (v3.3.0) package, Sequenza (v2.1.0). Significantly amplified and deleted regions were detected using GISTIC (v2.0.23). Gene-specific copy number calls were generated using a custom R script (See Supplementary Methods). Wholegenome duplication was inferred using code described by López S et al. ${ }^{45}$.

RNA in situ hybridization. Expressions of $B N L F 2 a, L M P 1$, and TGFBR2 transcripts were detected by RNAscope 2.0 RISH assays using BNLF2a, LMP1, and TGFBR2specific probes (Advanced Cell Diagnostics, USA) as previously described ${ }^{15,46}$. A RNAscope probe V-HHV4-BNLF2-C2 was used for the detection of BNLF2a transcripts while the probes V-HHV4-LMP1 and BA-V-EBV-LMP1-2EJ were used to confirm the presence of LMP1 transcription signals in the NPC specimens. The cases with LMP1 transcripts signals were excluded from $B N L F 2 a$ detection analysis because of the overlap of the BNLF2a gene and exon 3 of $L M P 1$. BNLF2a and TGFBR2 expression was assessed by assigning a proportion score and an intensity score. The proportion score relates to the percentage of tumor cells with a positive signal $(0-100)$. The intensity of the signal in the tumor cells was scored as 0 for none; 1 for weak, 2 for intermediate, and 3 for strong. The gene expression score was calculated as the product of the proportion and intensity scores, ranging from 0 to 300 .

Immunohistochemical staining. MHC-I, MHC-II, PDL1, LMP1, MTAP protein expressions were determined on formalin-fixed, paraffin-embedded sections by immunohistochemical staining. After de-waxing, the sections were subjected to antigen retrieval and staining by the automated slide processing system BenchMark XT (Ventana Medical System Inc., Tucson, AZ, USA) as previously published ${ }^{8,35}$. The primary antibody used in this study was anti-LMP1 mouse monoclonal antibody (diluted 1:1000, clone CS.1-4, Dako, Agilent Technologies, USA), antiPDL1 (22C3, Dako, Agilent Technologies, USA), anti-HLA Class I A/B/C (diluted 1:1000, clone ECR8-5, ab7038, Abcam, USA), anti-MHC Class II (diluted 1:2000, clone 6C6, ab55152, Abcam, USA), anti-MTAP (diluted 1:400; 4158, Cell Signaling, USA), anti-Involucrin (diluted 1:100, clone SY5, MA5-11803, Invitrogen, USA) and p53 (diluted 1:100, clone DO-1, SC-126, Santa Cruz, USA).

Immunoblotting. Total cell lysates extracted from the xenografts and cell lines were separated by sodium dodecyl sulphate-polyacrylamide gel electrophoresis and transferred to a polyvinylidene difluoride membrane for immunoblotting as described $^{41}$. Primary antibodies used in this study were anti-TGFBR2 (diluted 1:1000, clone D-2, sc-17799, Santa Cruz, USA), anti-SMAD2 (diluted 1:1000, clone D43B4, 5339, Cell Signaling, USA), anti-pSMAD2 (diluted 1:1000, clone 138D4, 3108 Cell Signaling, USA), anti-SMAD3 (diluted 1:1000, clone C67H9, 9523, Cell Signaling, USA), anti-pSMAD3 (diluted 1:1000, clone C25A9, 9520, Cell Signaling, USA), anti-Involucrin (diluted 1:1000, clone SY5, MA5-11803, Invitrogen, USA) anti-MTAP (diluted 1:1000, $4158 \mathrm{~S}$, Cell Signaling, USA), anti-PRMT5 (diluted 1:1000, 2252 S, Cell Signaling, USA), anti-MAT2A(diluted 1:10000, clone B-10, Sc166452, Santa Cruz, USA), anti-SDMA (diluted 1:1000, 13222 S, Cell Signaling, USA), anti-Caspase-3 (Asp175)(diluted 1:1000, clone 5A1E, 9664, Cell Signaling, USA), anti-BAX (diluted: 1000, 2772, Cell Signaling, USA), anti-p53 (diluted 1:1000, clone DO-1, Sc-126, Santa Cruz, USA), anti-Actin (diluted 1:100000, clone13E5, 4967, Cell Signaling, USA).

Quantitative polymerase chain reaction. Peripheral blood was collected from the treated mice. DNA was extracted from $100 \mu \mathrm{l}$ serum using QIAamp Blood mini kit (Qiagen) according to manufacturer's protocol and eluted in $20 \mu$ l elution buffer. Circulating EBV DNA was measured by quantitative PCR that amplified a region in the BamH1-W fragment of EBV genome ${ }^{47}$. Concentrations of EBV DNA were calculated using DNA extracted from EBV-positive Namalwa cells as standard and expressed as copies of EBV per $\mathrm{ml}$ serum. The primers and their sequences are shown in Supplementary data 7.

EBV infection of immortalized NPE cells. Immortalized NPE cells were infected with a GFP-tagged recombinant EBV (Akata strain) using an established protocol $^{31}$. After co-culturing of Akata cells and immortalized NPE cells for $48 \mathrm{~h}$, the infected epithelial cells were subjected to fluorescent-activated cell sorting (FACS) using a BD FACSARIA III system (BD biosciences, USA) to count and isolate GFP(EBV)-positive cells. The isolated EBV-positive cells were then cultured for 7 or 14 days. Growth media were replaced every $48 \mathrm{~h}$. At respective time points, cells were harvested and the percentage of EBV-positive cells was determined using the BD FACSARIA III system.

Cell proliferation assay. The cell proliferation reagent CCK- 8 was purchased from Dojindo Molecular Technologies (Rockville, MD, USA). In general, $5 \times 10^{3}$ cells in 100 $\mu \mathrm{l}$ of medium were seeded into 96-well plates in triplicate. The CCK-8 assay was performed as follows. In brief, $10 \mu \mathrm{l}$ of CCK- 8 reagent was added to each well and incubated at $37^{\circ} \mathrm{C}$ for $4 \mathrm{~h}$. The absorbance was then measured at $450 \mathrm{~nm}$. Each sample was performed in triplicate. The relative growth rate was calculated by dividing absorbance at indicated time points by the absorbance at day 1 after cell plating.

In vitro drug study. In general, $5 \times 10^{3}$ cells in $100 \mu \mathrm{l}$ of medium were seeded into 96-well plates in triplicate overnight and then treated with various concentrations of FIDAS-5 (Merck Millipore, USA) for $144 \mathrm{hrs}$. At the end of treatment, the medium was refreshed and $10 \mu \mathrm{l}$ of CCK- 8 reagent (Dojindo Molecular Technologies) was added to each well for assay as described above.

In vivo mouse experiments. Minced tumor tissues of PDX xeno-76 were subcutaneously inoculated into the flanks of 3-4-week-old female athymic mice with a starting weight of $\sim 18-22 \mathrm{~g}$ and allowed to grow to $\sim 50 \mathrm{~mm}^{3}$. Mice are kept within animal room limits of $20-23^{\circ} \mathrm{C}$ and $40-60 \%$ humidity. The mice run on a $12 \mathrm{~h} \mathrm{light/dark} \mathrm{cycle} \mathrm{that} \mathrm{from} 7$ am to $7 \mathrm{pm}$. Eight mice per group were used. FIDAS-5 (40 mg per $\mathrm{kg}$ ) or vehicle (corn oil) was administered via intraperitoneal injection once daily. Mice were weighed and tumors were measured with a caliper every 3 days. When the tumor sizes exceeded $1000 \mathrm{~mm}^{3}$, mice were killed, tumor and blood samples were collected for analysis. Tumor volume was calculated by the formula $0.5 \times l \times w^{2}$, where $l$ and $w$ are tumor length and width, respectively.

All animal care and experimental procedures were approved by the University Animal Experimentation Ethics Committee (AEEC), the Chinese University of Hong Kong. The animal license was obtained from the Hong Kong Government, Department of Health.

Reporting summary. Further information on research design is available in the Nature Research Reporting Summary linked to this article.

\section{Data availability}

The raw data in the BAM files for the WGS sequencing from this study have been deposited in European Genome-phenome Archive (EGA) under accession number: EGAS00001004705 in the hyperlink. Access to this data set in EGA can be requested from UHN Genomics Data Access Committee (Contact person: Natalie Stickle, Email: natalie.stickle.uhn.ca). Full mutation and copy number calls are included as part of the supplementary information and the remaining data are available from the authors upon request. A publicly available WGS data set from a cohort of 12 NPC samples deposited at the Sequence Read Archive (SRA, https://submit.ncbi.nlm.nih.gov/subs/sra/) was used for validation of MTAP deletion in NPC ${ }^{12}$ (https://doi.org/10.1093/carcin/bgy108). The accession codes of these NPC and corresponding normal blood samples are: SRR6431671, SRR6377819, SRR6431672, SRR6377820, SRR6431673, SRR6377821, SRR6431674, SRR6377822, SRR6431667, SRR6377823, SRR6431670, SRR6377824, SRR6431677, SRR6377825, SRR6431678, SRR6377826, SRR6431668, SRR6377827, SRR6431669, SRR6377828, SRR6431675, SRR6377829, SRR6431676, SRR6377830. In addition, simple somatic mutations and SV counts of human cancers reported by Campbell et al. 2017 (https://doi.org/10.1101/162784) were used for comparison with those detected in NPC samples in this study. The data set is available in web-link: https:// www.biorxiv.org/content/10.1101/162784v1.supplementary material. The NFKB1binding site motif of the significantly mutated non-coding variant (chr2: $10097565 \mathrm{C}>\mathrm{T}$ ) is available in: http://jaspar.genereg.net/matrix/MA0105.1/. Gencode v26 is available at: https://www.gencodegenes.org/human/release_26.html. Cosmic mutational signatures V2 (https://cancer.sanger.ac.uk/signatures/signatures_v2/) was used for determining the mutational signatures of NPC samples. Igenomes hg38 reference is available at: http:// igenomes.illumina.com.s3-website-us-east-1.amazonaws.com/Homo_sapiens/UCSC/ hg38/ Homo_sapiens_UCSC_hg38.tar.gz. Source data are provided with this paper.

Received: 6 October 2020; Accepted: 19 May 2021; Published online: 07 July 2021 


\section{References}

1. Lo, K. W., To, K. F. \& Huang, D. P. Focus on nasopharyngeal carcinoma. Cancer Cell 5, 423-428 (2004).

2. Tsang, C. M., Lui, V. W. Y., Bruce, J. P., Pugh, T. J. \& Lo, K. W. Translational genomics of nasopharyngeal cancer. Semin. Cancer Biol. 61, 84-100 (2020).

3. Tsao, S. W., Tsang, C. M., To, K. F. \& Lo, K. W. The role of Epstein-Barr virus in epithelial malignancies. J. Pathol. 235, 323-333 (2015).

4. Shannon-Lowe, C. \& Rickinson, A. The global landscape of EBV-associated tumors. Front. Oncol. 9, 713 (2019).

5. Giulino, L. et al. A20 (TNFAIP3) genetic alterations in EBV-associated AIDSrelated lymphoma. Blood 117, 4852-4854 (2011).

6. Kataoka, K. et al. Frequent structural variations involving programmed death ligands in Epstein-Barr virus-associated lymphomas. Leukemia 33, 1687-1699 (2019).

7. Cancer Genome Atlas Research Network. Comprehensive molecular characterization of gastric adenocarcinoma. Nature 513, 202-209 (2014).

8. Li, Y. Y. et al. Exome and genome sequencing of nasopharynx cancer identifies NF- $\kappa$ B pathway activating mutations. Nat. Commun. 8, 14121 (2017).

9. Lin, D. C. et al. The genomic landscape of nasopharyngeal carcinoma. Nat. Genet. 46, 866-871 (2014).

10. Zheng, $\mathrm{H}$. et al. Whole-exome sequencing identifies multiple loss-of-function mutations of NF- $\mathrm{kB}$ pathway regulators in nasopharyngeal carcinoma. Proc. Natl Acad. Sci. USA 113, 11283-11288 (2016).

11. Zhang, L. et al. Genomic Analysis of nasopharyngeal carcinoma reveals TMEbased subtypes. Mol. Cancer Res. 15, 1722-1732 (2017)

12. $\mathrm{Tu}, \mathrm{C}$. et al. Identification of genomic alterations in nasopharyngeal carcinoma and nasopharyngeal carcinoma-derived Epstein-Barr virus by whole-genome sequencing. Carcinogenesis 39, 1517-1528 (2018).

13. You, R. et al. Clonal mutations activate the NF-kB pathway to promote recurrence of nasopharyngeal carcinoma. Cancer Res. 79, 5930-5943 (2019).

14. Wang, F. H. Efficacy, safety, and correlative biomarkers of toripalimab in previously treated recurrent or metastatic nasopharyngeal carcinoma: a phase II clinical trial (POLARIS-02). J. Clin. Oncol. 39, 704-712 (2021).

15. Lin, W. et al. Establishment and characterization of new tumor xenografts and cancer cell lines from EBV-positive nasopharyngeal carcinoma. Nat. Commun. 9, 4663 (2018)

16. Dai, W. et al. Clinical outcome-related mutational signatures identified by integrative genomic analysis in nasopharyngeal carcinoma. Clin. Cancer Res. 26, 6494-6504 (2020).

17. Majdalawieh, A., Zhang, L. \& Ro, H. S. Adipocyte enhancer-binding protein-1 promotes macrophage inflammatory responsiveness by up-regulating NFkappaB via IkappaBalpha negative regulation. Mol. Biol. Cell. 18, 930-942 (2007).

18. Shah, K. N., Wilson, E. A., Malla, R., Elford, H. L. \& Faridi, J. S. Targeting ribonucleotide reductase $\mathrm{M} 2$ and NF- $\mathrm{kB}$ activation with didox to circumvent tamoxifen resistance in breast cancer. Mol. Cancer Ther. 14, 2411-2421 (2015).

19. Zhu, H. et al. Candidate cancer driver mutations in distal regulatory elements and long-range chromatin interaction networks. Mol. Cell 77, 1307-1321 (2020).

20. Or, Y. Y. et al. Identification of a novel $12 \mathrm{p} 13.3$ amplicon in nasopharyngeal carcinoma. J. Pathol. 220, 97-107 (2010)

21. Keats, J. J. et al. Promiscuous mutations activate the noncanonical NF-kappaB pathway in multiple myeloma. Cancer Cell 12, 131-144 (2007).

22. Annunziata, C. M. et al. Frequent engagement of the classical and alternative NF-kappaB pathways by diverse genetic abnormalities in multiple myeloma. Cancer Cell 12, 115-130 (2007).

23. Tsao, S. W., Tsang, C. M. \& Lo, K. W. Epstein-Barr virus infection and nasopharyngeal carcinoma. Philos. Trans. R. Soc. Lond. B Biol. Sci. 372, 20160270 (2017)

24. Jangra, S., Yuen, K. S., Botelho, M. G. \& Jin, D. Y. Epstein-Barr virus and innate immunity: friends or foes? Microorganisms 7, 183 (2019).

25. Hopcraft, S. E. \& Damania, B. Tumour viruses and innate immunity. Philos. Trans. R. Soc. Lond. B Biol. Sci. 372, 20160267 (2017).

26. Horst, D. et al. EBV protein BNLF2a exploits host tail-anchored protein integration machinery to inhibit TAP. J. Immunol. 186, 3594-3605 (2011).

27. Strong, M. J. et al. Latent expression of the Epstein-Barr virus (EBV)-encoded major histocompatibility complex class I TAP inhibitor, BNLF2a, in EBVpositive gastric carcinomas. J. Virol. 89, 10110-10114 (2015).

28. Ikushima, H. \& Miyazono, K. TGFbeta signalling: a complex web in cancer progression. Nat. Rev. Cancer 10, 415-424 (2010).

29. Velapasamy, S., Dawson, C. W., Young, L. S., Paterson, I. C. \& Yap, L. F. The dynamic roles of TGF- $\beta$ signalling in EBV-associated cancers. Cancers 10, E247 (2018).

30. Chan, A. S. et al. High frequency of chromosome $3 p$ deletion in histologically normal nasopharyngeal epithelia from southern Chinese. Cancer Res. 60, 5365-5370 (2000).
31. Tsang, C. M. et al. Cyclin D1 overexpression supports stable EBV infection in nasopharyngeal epithelial cells. Proc. Natl. Acad. Sci. USA 109, E3473-E3482 (2012).

32. Nowell, C. S. \& Radtke, F. Notch as a tumour suppressor. Nat. Rev. Cancer 17, 145-159 (2017).

33. Roemer, M. G. et al. Major histocompatibility complex class II and programmed death ligand 1 expression predict outcome after programmed death 1 blockade in classic hodgkin lymphoma. J. Clin. Oncol. 36, 942-950 (2018).

34. Hsu, C. et al. Safety and antitumor activity of Pembrolizumab in patients with programmed death-ligand 1-positive nasopharyngeal carcinoma: results of the KEYNOTE-028 study. J. Clin. Oncol. 35, 4050-4056 (2017).

35. Ma, B. B. et al. Antitumor activity of Nivolumab in recurrent and metastatic nasopharyngeal carcinoma: an international, multicenter study of the Mayo Clinic Phase 2 Consortium (NCI-9742). J. Clin. Oncol. 36, 1412-1418 (2018).

36. Fang, W. et al. Camrelizumab (SHR-1210) alone or in combination with gemcitabine plus cisplatin for nasopharyngeal carcinoma: results from two single-arm, phase 1 trials. Lancet Oncol. 19, 1338-1350 (2018).

37. Mavrakis, K. J. et al. Disordered methionine metabolism in MTAP/CDKN2Adeleted cancers leads to dependence on PRMT5. Science 351, 1208-1213 (2016).

38. Kryukov, G. V. et al. MTAP deletion confers enhanced dependency on the PRMT5 arginine methyltransferase in cancer cells. Science 351, 1214-1218 (2016).

39. Marjon, K. et al. MTAP Deletions in cancer create vulnerability to targeting of the MAT2A/PRMT5/RIOK1 axis. Cell Rep. 15, 574-587 (2016).

40. Kalev, P. et al. MAT2A inhibition blocks the growth of MTAP-deleted cance cells by reducing PRMT5-dependent mRNA splicing and inducing DNA damage. Cancer Cell 39, 209-224 (2021).

41. Chung, G. T. et al. Constitutive activation of distinct NF- $\mathrm{kB}$ signals in EBVassociated nasopharyngeal carcinoma. J. Pathol. 231, 311-322 (2013).

42. Wang, C. et al. Genome-wide CRISPR-based gene knockout screens reveal cellular factors and pathways essential for nasopharyngeal carcinoma. J. Biol. Chem. 294, 9734-9745 (2019)

43. Chan, S. Y. et al. Authentication of nasopharyngeal carcinoma tumor lines. Int. J. Cancer 122, 2169-2171 (2008).

44. Bernheim, A., Rousselet, G., Massaad, L., Busson, P. \& Tursz, T. Cytogenetic studies in three xenografted nasopharyngeal carcinomas. Cancer Genet. Cytogenet. 66, 11-15 (1993).

45. López, S. et al. Interplay between whole-genome doubling and the accumulation of deleterious alterations in cancer evolution. Nat. Genet. 52, 283-293 (2020).

46. Lee, H. M. et al. Oncogenic S1P signalling in EBV-associated nasopharyngeal carcinoma activates AKT and promotes cell migration through S1P receptor 3. J. Pathol. 242, 62-72 (2017)

47. Lo, Y. M. et al. Quantitative analysis of cell-free Epstein-Barr virus DNA in plasma of patients with nasopharyngeal carcinoma. Cancer Res. 59, 1188-1191 (1999).

\section{Acknowledgements}

T.J.P. was supported by the Princess Margaret Cancer Foundation; Canada Research Chairs program; Canada Foundation for Innovation, Leaders Opportunity Fund, CFI \#32383; and the Ontario Ministry of Research and Innovation, Ontario Research Fund Small Infrastructure Program. K.W.L. was supported by Research Grant Council, Hong Kong (Areas of Excellence Scheme - AoE/M-401/20; Theme-based Research Scheme T12-401/13-R; Collaborative Research Fund - C4001-18GF, C7027- 16 G, C5012-15E; General Research Fund - 14104415, 14138016, 14117316, 14101721), Core Utilities of Cancer Genome and Pathobiology, Focused Innovations Scheme and Faculty Strategic Research (4620513) of the Faculty of Medicine, and VC's One-off Discretionary Fund (VCF2014017, VCF2014015), the Chinese University of Hong Kong. C.M.T. was supported by the Health and Medical Research Fund (05162386, 13142201), General Research Fund (14113620, 17110315), Faculty Innovation Award (FIA2020/A/01) and NSFC/RGC Joint Research Scheme (N_HKU735/18 and 81861168033) of Research Grant Council, Hong Kong. S.W.T. was supported by Research Grant Council Hong Kong (CRF-C7027-16G; GRF-17116416, GRF-17104617, GRF-17111917, GRF17114818, GRF-17122420, NSFC/RGC-N_HKU735/18 and 81861168033). L.F.Y. was supported by Fundamental Research Grant Scheme (FRGS/1/2019/SKK08/UM/02/6) from the Ministry of Higher Education Malaysia, Newton-Ungku Omar Fund MR/ P013201 (IF016-2017) from the Academy of Sciences Malaysia and Medical Research Council UK, and University of Malaya Impact-Oriented Interdisciplinary Research Grant Programme (IIRG008A-19FNW). V.W.Y.L. was supported by the Research Grant Council, Hong Kong (General Research Fund - 17114814, 17121616, 14168517; Research Impact Fund - R4015-19F, R4017-18), the Health and Medical Research Fund (HMRF\#15160691) from the Food and Health Bureau, The Government of the Hong Kong SAR; University-Industry Collaboration Program (UIM/329; Innovation and Technology Fund, Hong Kong government, Hong Kong SAR and Lee's Pharmaceutical 
(HK) Limited); the Hong Kong Cancer Fund, Hong Kong SAR; Start-up Fund from the School of Biomedical Sciences, Faculty of Medicine, CUHK.

\section{Author contributions}

K.W.L. and T.J.P. have led the study, designed and conducted the experiments, analyzed the data, interpreted the results, written the initial draft, and created the figures with the input of the senior authors J.P.B., K.F.T., V.W.Y.L. and G.T.C. who contributed equally. The following authors were involved in the genome sequencing, cell-based and in vivo experiments, developed methodologies, analyzed clinical samples, helped writing the manuscript, and approved the final version: Y.Y.C., C.M.T., B.B.Y.M., J.K.S.W., E.P.H., M.K.F.M., C.C., S.V., Y.Y.Y.O., P.K.S., J.Y.K.C., I.C.P., L.F.Y., C.W.D. The following authors analyzed the genome sequencing data, developed methodologies and/or helped with the interpretation of data helped writing the manuscript, and approved the final version: K.Y.Y., S.D.L., S.E.G., S.P. M.W., J.S.H.K., L.Y., S.W.T., L.S.Y., F.F.L. and A.T.C.C. supervised the research, provided material or funding support, helped writing the manuscript and approved the final version.

\section{Competing interests}

All authors declare no competing interests directly related to the current study. V.W.Y. Lui receives funding from a University-Industry Collaboration Program (UIM/329) by the Innovation and Technology Fund, Hong Kong government, and Lee's Pharmaceutical (Hong Kong Limited) for the period of 2018-2020, and also served as a scientific consultant for Novartis Pharmaceutical (Hong Kong) Limited (Oct 2015-Oct 2016). B.B. Y. Ma received speaker's honorarium and serves in the advisory board of Bristol-Myers Squibb (BMS), MSD and Novartis, Hong Kong. She has received a research grant from Novartis and Boerhinger Ingelheim. E.P. Hui received speaker's honoraria and serves in the advisory board of Merck Sharp \& Dohme (MSD) and Merck Serono. A.T.C. Chan received research and travel grants from MSD, Pfizer, and Roche. The other authors declare no competing interests.

\section{Additional information}

Supplementary information The online version contains supplementary material available at https://doi.org/10.1038/s41467-021-24348-6.

Correspondence and requests for materials should be addressed to T.J.P. or K.-W.L.

Peer review information Nature Communications thanks Erik Flemington and the other, anonymous reviewer(s) for their contribution to the peer review of this work. Peer review reports are available.

Reprints and permission information is available at http://www.nature.com/reprints

Publisher's note Springer Nature remains neutral with regard to jurisdictional claims in published maps and institutional affiliations.

\section{(c) (i)}

Open Access This article is licensed under a Creative Commons Attribution 4.0 International License, which permits use, sharing, adaptation, distribution and reproduction in any medium or format, as long as you give appropriate credit to the original author(s) and the source, provide a link to the Creative Commons license, and indicate if changes were made. The images or other third party material in this article are included in the article's Creative Commons license, unless indicated otherwise in a credit line to the material. If material is not included in the article's Creative Commons license and your intended use is not permitted by statutory regulation or exceeds the permitted use, you will need to obtain permission directly from the copyright holder. To view a copy of this license, visit http://creativecommons.org/ licenses/by/4.0/.

(C) The Author(s) 2021, corrected publication 2022 